\title{
OFDM-Based Turbo-Coded Hierarchical and Non-Hierarchical Terrestrial Mobile Digital Video Broadcasting
}

\author{
Chee-Siong Lee, Thoandmas Keller, and Lajos Hanzo, Senior Member, IEEE
}

\begin{abstract}
The feasibility of terrestrial Digital Video Broadcast (DVB) to mobile receivers is studied and turbo coded performance enhancements are proposed. Initially, the MPEG-2 codec is subjected to a rigorous bit error sensitivity investigation, in order to assist in designing various error protection schemes for wireless DVB transmission. The turbo codec is shown to provide Signal-to-Noise Ratio (SNR) performance advantages in excess of 5-6 dB over conventional convolutional coding both in terms of bit error rate and video quality. Our experiments suggested that-despite our expectations-multi-class data partitioning did not result in error resilience improvements, since a high proportion of relatively sensitive video bits had to be relegated to the lower integrity subchannel, when invoking a powerful low-rate channel codec in the high-integrity protection class. Nonetheless, DVB transmission to mobile receivers is feasible, when using turbo-coded OFDM transceivers at realistic power-budget requirements under the investigated highly dispersive fading channel conditions. It is interesting to note furthermore that the 5-6 dB SNR improvement due to turbo coding allows us to invoke for example the double-throughput 16-level Quadrature Amplitude Modulation (16-QAM) mode instead of the standard convolutional-coded 4-QAM mode. This facilitates doubling the bit rate and hence improving the video quality.
\end{abstract}

Index Terms-DVB, DVB-T, error sensitivity, hierarchical video transmission, mobile video broadcast, MPEG-2, OFDM, QAM, terrestrial video broadcast, wireless video broadcast.

\section{BACKGROUND AND MOTIVATION}

$\mathbf{F}$ OLLOWING the standardization of the Pan-European Digital Video Broadcasting (DVB) systems, we have begun to witness the arrival of digital television services to the home. However, for a high proportion of business and leisure travellers it is desirable to have access to DVB services while on the move. Although it is feasible to access these services with the aid of dedicated DVB receivers, these receivers may also find their way into the laptop computers of the near future. These intelligent laptops may also become the portable DVB receivers of wireless in-home networks.

In recent years three DVB standards have emerged in Europe for terrestrial [1], cable-based [2], and satellite-oriented [3] delivery of DVB signals. The more hostile propagation

Manuscript received July 7, 1999; revised February 24, 2000. This work was supported in part by EPSRC, U.K. in the framework of the Contract GR/K 74043 and the European Commission.

The authors are with the Department of Electronics and Computer Science, University of Southampton, SO17 1BJ, U.K. (e-mail: 1h@ecs.soton.ac.uk).

Publisher Item Identifier S 0018-9316(00)03980-9. environment of the terrestrial system requires concatenated Reed-Solomon [4], [5] (RS) and rate compatible punctured convolutional coding [4], [5] (RCPCC) combined with Orthogonal Frequency Division Multiplexing (OFDM) based modulation [6]. By contrast, the more benign cable and satellite based media facilitate the employment of multi-level modems using up to 256-level quadrature amplitude modulation (QAM) [6]. These schemes are capable of delivering high-definition video at bitrates of up to $20 \mathrm{Mbits} / \mathrm{s}$ in stationary broadcast-mode distributive wireless scenarios.

Recently, there has been a range of DVB system performance studies in the literature [7]-[10]. Against this background, in this contribution we have proposed turbo-coding based improvements to the terrestrial DVB system [1] and investigated its performance under hostile mobile channel conditions. We have also studied various video bitstream partitioning and channel coding schemes both in the so-called hierarchical and nonhierarchical transceiver modes to be discussed during our further discourse and compared their performance.

The rest of the paper is divided into the following sections. In Section II the bit error sensitivity of the MPEG-2 coding parameters [11] is characterized. A brief overview of the enhanced turbo-coded and standard DVB terrestrial scheme is presented in Section III, while the channel model is described in Section IV. Following this, in Section V the reader is introduced to the MPEG-2 data partitioning scheme [12] used to split the input MPEG-2 video bitstream into two error protection classes, which can then be protected either equally or unequally. These two video bit protection classes can then be broadcasted to the receivers using the so-called DVB terrestrial hierarchical transmission format [1]. The performance of the data partitioning scheme was investigated by corrupting either the high or low sensitivity video bits using randomly distributed errors for a range of system configurations in Section VI and their effects on the overall reconstructed video quality were evaluated. Following this, the performance of the improved DVB terrestrial system employing the so-called nonhierarchical and hierarchical format [1] is examined in a mobile environment in Sections VII and VIII, before our conclusions and future work areas are presented in Section IX. We note furthermore that readers mainly interested in the overall system performance may opt for directly proceeding to Section III. Let us commence our discourse in the next section by describing an objective method of quantifying the sensitivity of the MPEG-2 video parameters. 


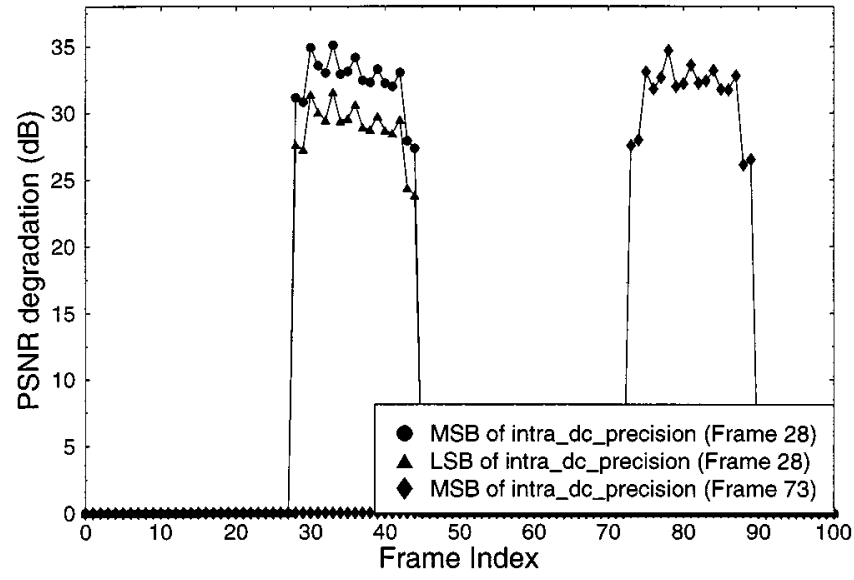

Fig. 1. PSNR degradation profile for the different bits used to encode the so-called intra_dc_precision parameter [11] in different corrupted video frames for the "Miss America" QCIF video sequence encoded at $30 \mathrm{frame} / \mathrm{s}$ and 1.15 Mbit/s.

\section{MPEG-2 BIT ERROR SENSITIVITY}

At this stage we have to note again that a number of different techniques can be used in order to quantify the bit error sensitivity of the MPEG-2 bits. The outcome of these investigations will depend to a degree on the video material used, the output bit rate of the video codec, the objective video quality measures used and the averaging algorithm employed. Perceptually motivated, subjective quality based sensitivity testing becomes simply infeasible due to the large number of associated test scenarios. Hence in this section a simplified objective video quality measure based bit-sensitivity evaluation procedure is proposed, which attempts to take into account all the major factors influencing the sensitivity of MPEG-2 bits. Specifically, the proposed procedure takes into account the position and the relative frequency of the MPEG-2 parameters in the bitstream, the number of the associated coding bits for each MPEG-2 parameter, the video bit rate and the effect of loss of synchronization or error propagation due to corrupted bits. Nonetheless, we note that a range of similar bit sensitivity estimation techniques exhibiting different strengths and weaknesses can be devised and no doubt future research will produce a variety of similarly motivated techniques.

In this section we assume familiarity with the MPEG-2 standard [11], [12]. The aim of our MPEG-2 error resilience study was to quantify the average PSNR degradation inflicted by each erroneously decoded video codec parameter in the bitstream, so that appropriate protection can be assigned to each parameter. First, we will define three measures, namely the peak signal-to-noise ratio (PSNR), the PSNR degradation and the average PSNR degradation, which are to be used in our subsequent discussions. The PSNR is defined as follows:

$$
\text { PSNR }=10 \log _{10} \frac{\sum_{n=0}^{N} \sum_{m=0}^{M} 255^{2}}{\sum_{n=0}^{N} \sum_{m=0}^{M} \Delta^{2}},
$$

where $\Delta$ is the difference between the uncoded pixel value and the reconstructed pixel value, while the variables $M$ and $N$ refer to the dimension of the image. The maximum possible 8-bit pixel luminance value of 255 was used in (1) in order to mitigate the PSNR's dependence on the video material used. The PSNR degradation is the difference between the PSNR of the decoder's reconstructed image in the event of erroneous decoding and successful decoding. The average PSNR degradation is then the mean of the PSNR degradation values computed for all the image frames of the video test sequence.

Most MPEG-2 parameters are encoded by several bits and they may occur in different positions in the video sequence. In these different positions they typically affect the video quality differently, since corrupting a specific parameter of a frame close to the commencement of a new picture start code inflicts a lesser degradation, than corrupting an equivalent parameter further from the resynchronization point. Hence the sensitivity of the MPEG-2 parameters is position-dependent. Furthermore, different encoded bits of the same specific MPEG-2 parameter may exhibit different sensitivity to channel errors. Fig. 1 shows such an example for the parameter known as intra_dc_precision [11], which is coded under the so-called Picture Coding Extension [12]. In this example, the PSNR degradation profiles due to bit errors being inflicted on the parameter intra_dc_precision of Frame 28 showed that the degradation is dependent on the significance of the bit considered. Specifically, errors in the most significant bit (MSB) caused an approximately $3 \mathrm{~dB}$ higher PSNR degradation, than the least significant bit (LSB) errors. Furthermore, the PSNR degradation due to a MSB error of the intra_dc_precision parameter in Frame 73 is similar to the PSNR degradation profile for the MSB of the intra_dc_precision parameter of Frame 28. Due to the variation of the PSNR degradation profile for the bits of different significance of a particular parameter, as well as for the same parameter at its different occurrences in the bitstream, it is necessary to determine the average PSNR degradation for each parameter in the MPEG-2 bitstream.

Our approach in obtaining the average PSNR degradation was similar to that suggested in [13] and [14]. Specifically, the average measure used here takes into account the significance of the bits corresponding to the MPEG-2 parameter concerned, as well as the occurrence of the same parameter at different locations in the encoded video bitstream. In order to find the average PSNR degradation for each MPEG-2 bitstream parameter, the different bits encoding a specific parameter, as well as the bits of the same parameter but occurring at different locations in the MPEG-2 bitstream were corrupted and the associated PSNR degradation profile versus frame index was registered. The observed PSNR degradation profile generated for different locations of a specific parameter was then used to compute the average PSNR degradation. As an example, we shall use the PSNR degradation profile shown in Fig. 1. In this figure there are three degradation profiles. The average PSNR degradation for each profile is first computed in order to produce three average PSNR degradation values corresponding to the three respective profiles. The mean of these three PSNR averages will then form the final average PSNR degradation for the intra_dc_precision parameter. The same process is repeated for all MPEG-2 param- 


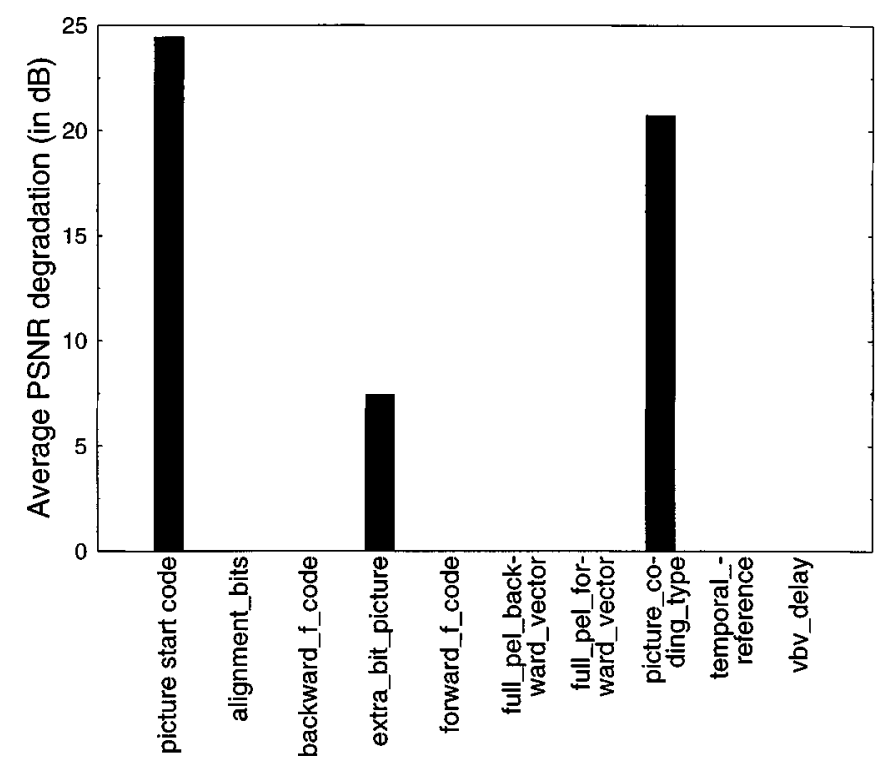

(a)

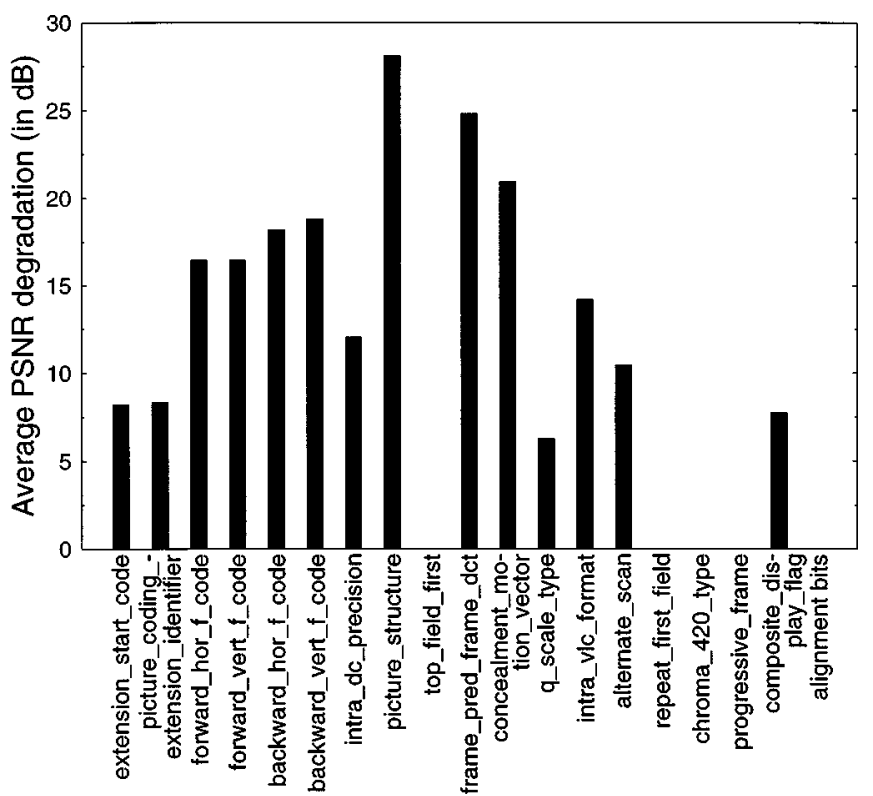

(b)

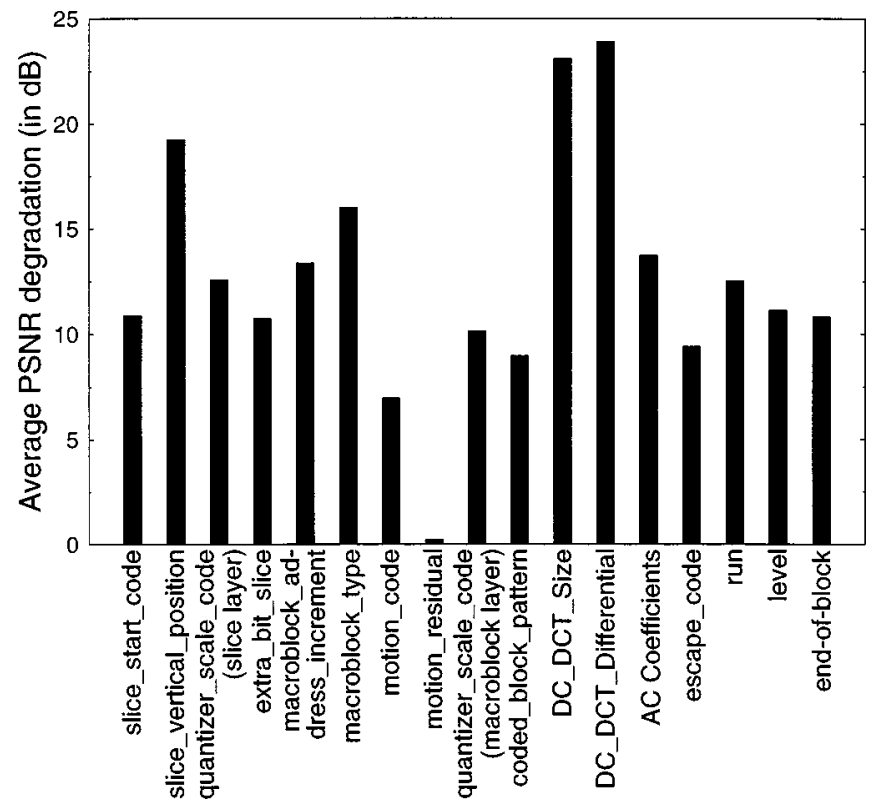

(c)

Fig. 2. Average PSNR degradation for the various MPEG-2 parameters in (a) Picture Header Information, (b) Picture Coding Extension, and (c) Slice-, Macroblock-, and Block-Layers for the "Miss America" QCIF video sequence encoded at $30 \mathrm{frame} / \mathrm{s}$ and 1.15 Mbit/s.

eters from the Picture Layer up to the Block Layer. The difference with respect to the approach adopted in [13], [14] was that while in [13], [14] the average PSNR degradation was acquired for each bit of the output bitstream, we have adopted a simpler approach in this contribution due to the large number of different parameters within the MPEG-2 bitstream. Fig. 2 shows the typical average PSNR degradations of the various MPEG-2 parameters of the Picture Header Information, Picture Coding Extension, Slice Layer, Macroblock Layer, and Block Layer [12], which were obtained using the $176 \times 144$ quarter common intermediate format (QCIF) "Miss America" (MA) video sequence at $30 \mathrm{frames} / \mathrm{s}$ and a high average bitrate of $1.15 \mathrm{Mbits} / \mathrm{s}$.

However, the different MPEG-2 parameters or codewords occur with different probabilities and they are allocated different numbers of bits. Therefore, the average PSNR degradation registered in Fig. 2 for each MPEG-2 parameter was multiplied with the long-term probability of this MPEG-2 parameter occurring in the MPEG-2 bitstream and with the relative probability of bits being allocated to that MPEG-2 parameter. Figs. 3 and 4 show the probability of occurrence of the various MPEG-2 parameters characterized in Fig. 2 and the probability of bits allocated to the parameters in the Picture Header Information, Picture Coding Extension, as well as in the Slice-, Macroblock- and Block-Layers [12], respectively, for the QCIF MA video sequence encoded at $1.15 \mathrm{Mbit} / \mathrm{s}$.

We shall concentrate first on Fig. 3(a). It is observed that all parameters-except for the full_pel_forward_vector, forward_f_code, full_pel_backward_vector, and back- 


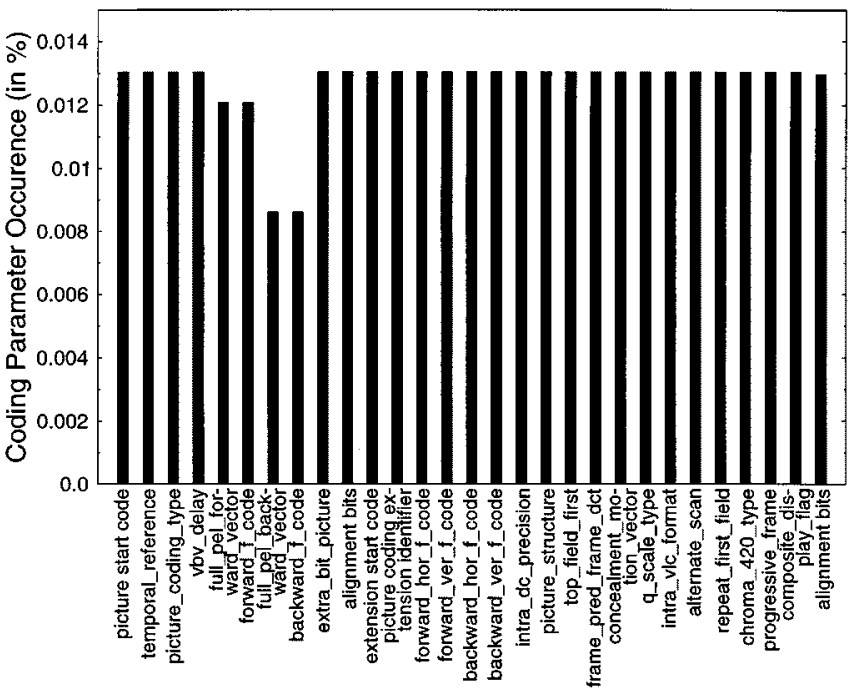

(a)

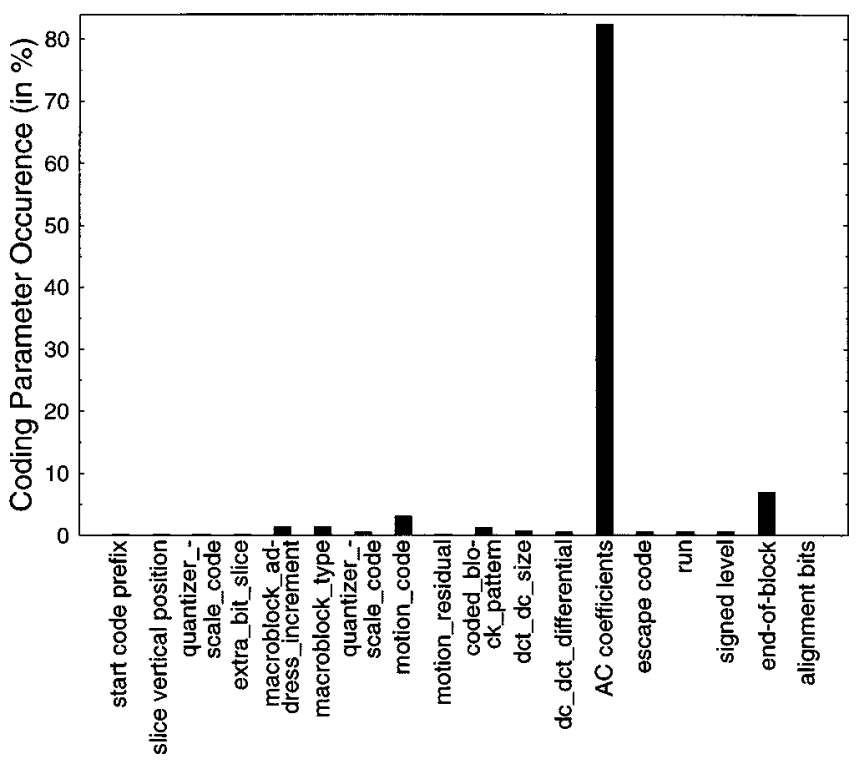

(b)

Fig. 3. Probability of occurrence for the various MPEG-2 parameters characterized in Fig. 2(a) Picture Header Information and Picture Coding Extension and (b) Slice-, Macroblock-, and Block-Layers for the "Miss America" QCIF video sequence encoded at $30 \mathrm{frame} / \mathrm{s}$ and $1.15 \mathrm{Mbit} / \mathrm{s}$.

ward_f_code_have the same probability of occurrence, since they appear once for every coded video frame. The parameters full_pel_forward_vector and forward_f_code have a higher probability of occurrence than full_pel_backward_vector and backward_f_code, since the former two appear in both $P$-frames and $B$-frames, while the latter two only occur in $B$-frames. For our experiments, the MPEG-2 encoder was configured such that for every encoded $P$-frame, there were two encoded $B$-frames. However, when compared with the parameters from the Slice-Layer, Macroblock-Layer and Block-Layer, which are characterized by the bar chart of Fig. 3(b), the parameters of the Picture Header Information and Picture Coding Extension appeared significantly less frequently.

If we compare the frequency of occurrence of the parameters in the Slice-Layer with those in the Macroblock- and BlockLayers, the former appeared less often, since there were 11 mac- roblocks and 44 blocks per video frame slice for the QCIF Miss America video sequence considered in our experiments. The AC Discrete Cosine Transform (DCT) [15] coefficient parameter had the highest probability of occurrence, exceeding eighty percent.

Fig. 4 shows the probability of bits being allocated to the various MPEG-2 parameters in the Picture Header Information, Picture Coding Extension, Slice-, Macroblock-, and Block-Layers [12]. Fig. 5 was included to more explicitly illustrate the probability of bit allocation seen in Fig. 4(b), with the probability of allocation of bits to the AC DCT coefficients being omitted from the bar-chart. Considering Fig. 4(a), the two dominant parameters, with the highest number of encoding bits requirement, are the picture start code (PSC) and the picture coding extension start code (PCESC). However, comparing these probabilities with the probability of bits being allocated to the various parameters in the Slice-, Macroblock-, and Block-Layers, the percentage of bits allocated can still be considered minimal due to their infrequent occurrence. In the Block-Layer, the AC DCT coefficients require in excess of 85 percent of the bits available for the whole video sequence. However, at bit rates lower than $1.15 \mathrm{Mbit} / \mathrm{s}$ the proportion of AC-coefficient encoding bits was significantly reduced, as illustrated by Fig. 6. Specifically, at 30 frames/s and 1.15 $\mathrm{Mbit} / \mathrm{s}$, the average number of bits per video frame is about 38000 and a given proportion of these bits is allocated to the MPEG-2 control header information, motion information and to the DCT coefficients. Upon reducing the total bitrate budget - since the number of control header bits is more or less independent of the target bitrate - the proportion of bits allocated to the DCT coefficients is substantially reduced. This is explicitly demonstrated in Fig. 6 for bit rates of $1.15 \mathrm{Mbit} / \mathrm{s}$ and $240 \mathrm{kbit} / \mathrm{s}$ for the "Miss America" QCIF video sequence.

The next process, as discussed earlier, was to normalize the measured average PSNR degradation according to the probability of occurrence of the respective MPEG-2 parameters in the bitstream and the probability of bits being allocated to this parameter. The normalized average PSNR degradation caused by corrupting the parameters of the Picture Header Information and Picture Coding Extension [12] is portrayed in Fig. 7(a). Similarly, the normalized average PSNR degradation for the parameters of the Slice-, Macroblock-, and Block-Layers is shown in Fig. 7(b). In order to visually enhance Fig. 7(b), the normalized average PSNR degradation for the AC DCT coefficients was omitted in the bar-chart shown in Fig. 8.

The highest PSNR degradation was inflicted by the AC DCT coefficients, since these parameters occur most frequently and hence are allocated the highest number of bits. When a bit error occurs in the bitstream, the AC DCT coefficients have a high probability of being corrupted. The other parameters, such as the DC_DCT_size and DC_DCT_differential, though exhibiting high average PSNR degradations when corrupted, registered low normalized average PSNR degradations since their occurrence in the bitstream is confined to the infrequent intra-coded frames.

The end-of-block MPEG-2 parameter exhibited the second highest normalized average PSNR degradation in this study. Although the average number of bits used for the end-of-block is 


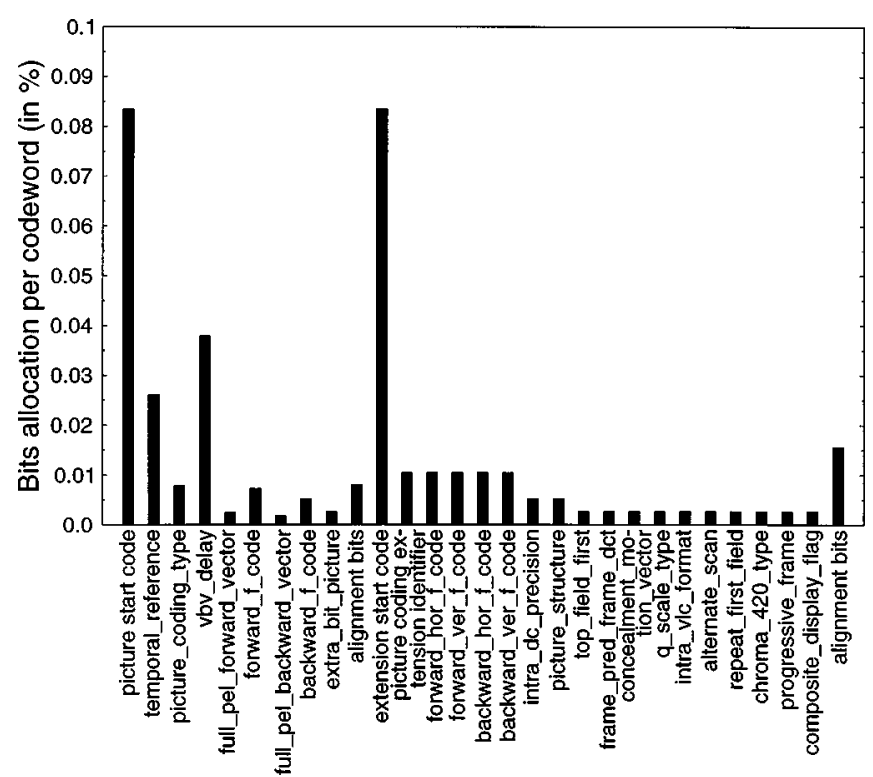

(a)

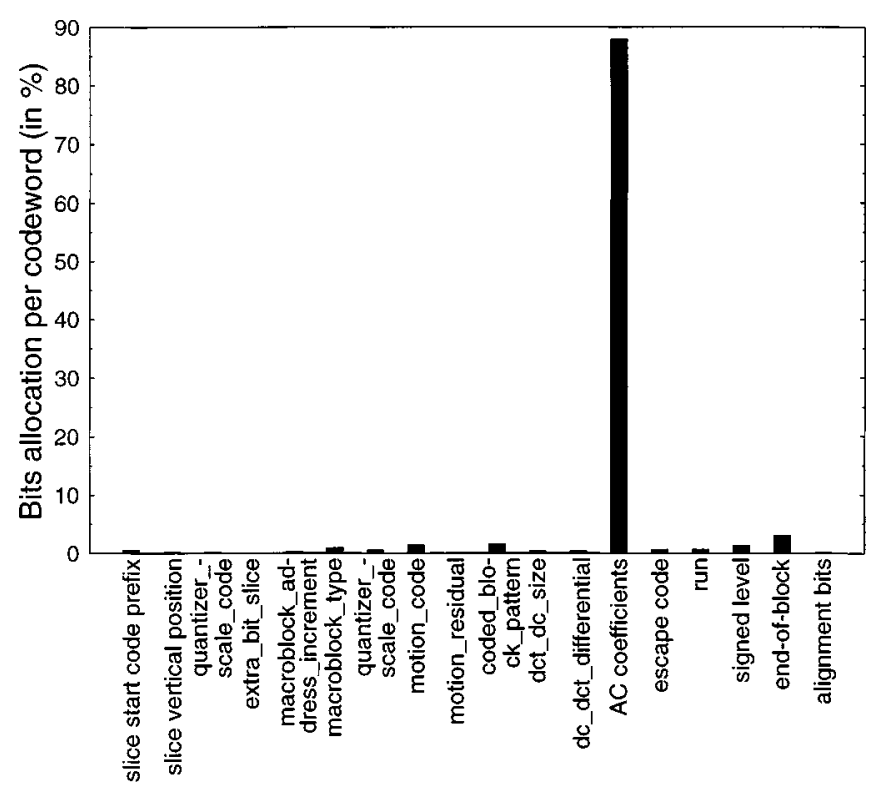

(b)

Fig. 4. Probability of bits being allocated to parameters in (a) Picture Header Information and Picture Coding Extension (b) Slice-, Macroblock-, and Block-Layers for the "Miss America" QCIF video sequence encoded at 30 frame/s and $1.15 \mathrm{Mbit} / \mathrm{s}$.

only approximately 2.17 bits, the probability of occurrence and the probability of bits being allocated to it is higher than those of other parameters, with the exception of the AC DCT coefficients. Furthermore, in general, the parameters of the Slice-, Macroblock-, and Block-Layers exhibit higher average normalized PSNR degradations due to their more frequent occurrence in the bitstream compared to the parameters, which belong to the Picture Header Information and to the Picture Coding Extension. This also implies that the percentage of bits allocated to these parameters is higher.

Comparing the normalized average PSNR degradations of the parameters in the Picture Header Information and Picture Coding Extension, the picture start code (PSC) exhibits the

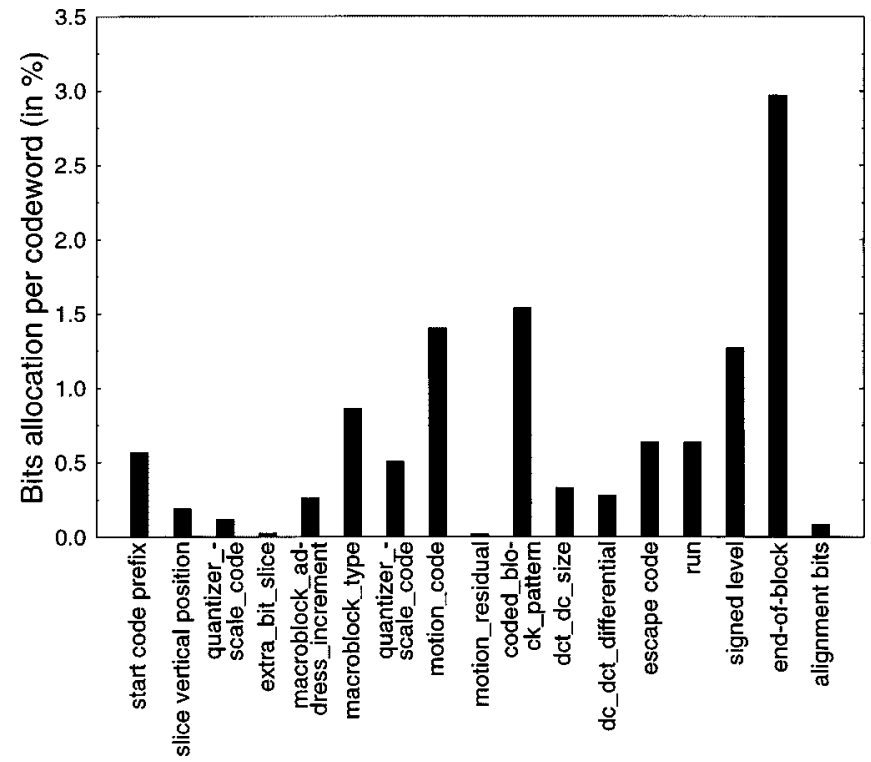

Fig. 5. Probability of bits being allocated to the various MPEG-2 Slice-, Macroblock-, and Block-Layer parameters, as seen in Fig. 4(b), where the probability of bits allocated to the AC DCT coefficients was omitted, in order to show the allocation of bits to the other parameters more clearly. This probability of bits allocation to the various MPEG-2 parameters is associated with the "Miss America" QCIF video sequence encoded at 30 frame/s and 1.15 Mbit/s.

highest normalized average PSNR degradation. Although most of the parameters here occur with equal probability as seen in Fig. 3(a), the picture start code requires a higher portion of the bits compared to the other parameters, with the exception of the extension start code. Despite having the same probability of occurrence and the same allocation of bits, the extension start code exhibits a lower normalized PSNR degradation than the picture start code, since its average un-normalized degradation is lower, as shown in Fig. 2.

From Figs. 7 and 8, we observe that the video PSNR degradation was dominated by the erroneous decoding of the AC DCT coefficients, which appeared in the MPEG-2 video bitstream in the form of variable length codewords. This suggests invoking unequal error protection techniques for protecting the MPEG-2 parameters during transmission. In a low complexity implementation, two protection classes may be envisaged. The higher priority class would contain all the important header information and some of the more important low-frequency variable-length coded DCT coefficients. The lower priority class would then contain the remaining less important, higher frequency variable length coded DCT coefficients. This partitioning process will be detailed in Section V together with its associated performance in the context of the hierarchical DVB [1] transmission scheme in Section VIII. Let us, however, first consider the architecture of the investigated DVB system in the next section.

\section{DVB TERRESTRIAL SCHEME}

The block diagram of the DVB terrestrial (DVB-T) transmitter [1] is shown in Fig. 9, which consists of an MPEG-2 video encoder, channel coding modules and an Orthogonal Frequency Division Multiplex (OFDM) modem [6], [16]. The bitstream 


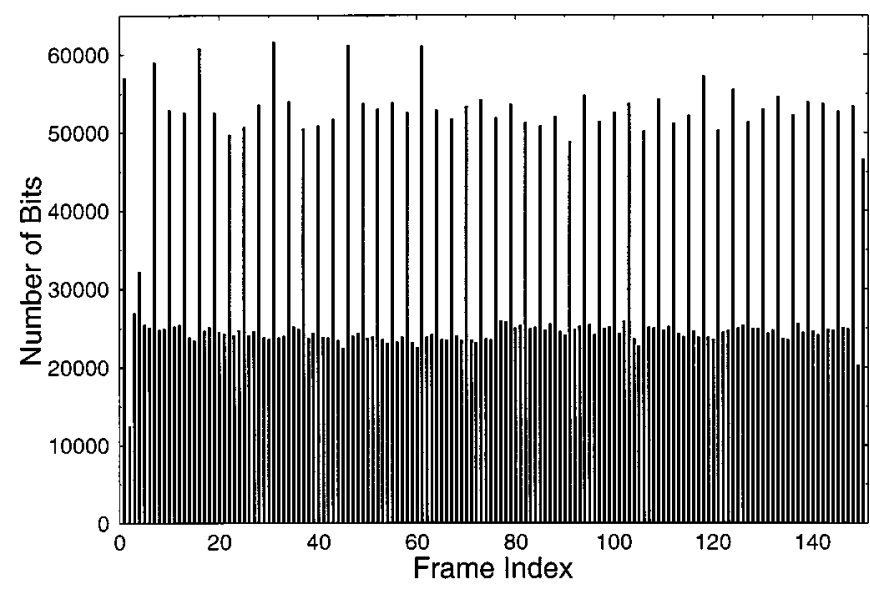

(a)

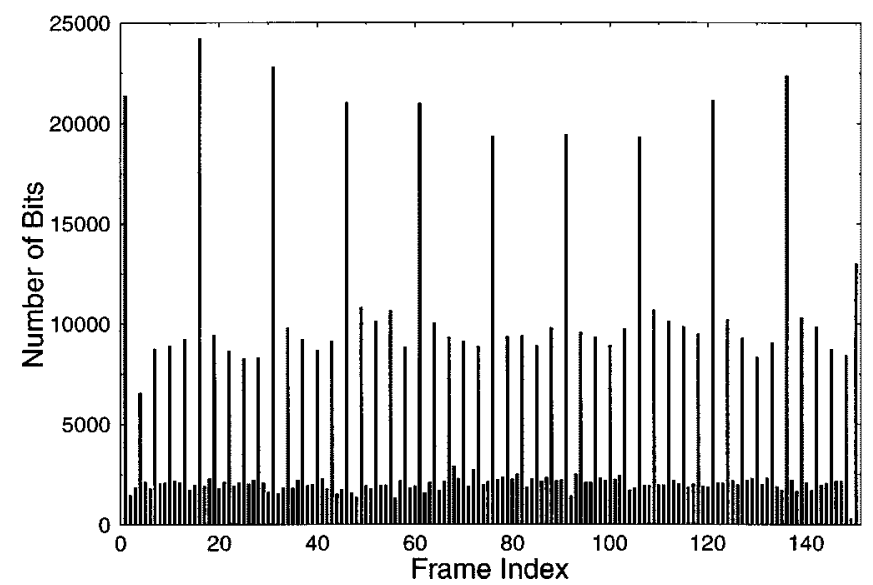

(b)

Fig. 6. Profile of bits allocated to the DCT coefficients, when the 30 frame/s QCIF Miss America video sequence is coded at (a) $1.15 \mathrm{Mbit} / \mathrm{s}$ (top) and (b) $240 \mathrm{kbit} / \mathrm{s}$ (bottom). The sequence of frames is in the order $I B B, P B B, P B B, P B B$ and so on.

generated by the MPEG-2 encoder is packetized into frames of 188 -byte long. The video data in each packet is then randomized by the scrambler of Fig. 9 . The specific details concerning the scrambler have not been included in this paper, since these may be obtained from the DVB-T standard [1].

Due to the poor error resilience of the MPEG-2 video codec, powerful concatenated channel coding is employed. The concatenated channel codec of Fig. 9 comprises a shortened Reed-Solomon (RS) outer code and an inner convolutional encoder. The 188-byte MPEG-2 video packet is extended by the Reed-Solomon encoder [4], [5] with parity information to facilitate error recovery in order to form a 204-byte packet. The Reed-Solomon decoder can then correct up to eight erroneous bytes for each 204-byte packet. Following this, the RS-coded packet is interleaved by a convolutional interleaver and further protected by a half-rate inner convolutional encoder using a constraint length of 7 [4], [5].

Furthermore, the overall code rate of the concatenated coding scheme can be adapted by variable puncturing which supports code rates of $1 / 2$ (no puncturing) as well as $2 / 3,3 / 4,5 / 6$, and $7 / 8$. The parameters of the convolutional encoder are summarized in Table I.
If only one of the two branches of the transmitter in Fig. 9 is utilized, the DVB-T modem is said to be operating in its nonhierarchical mode. In this mode, the modem can have a choice of QPSK, 16-QAM, or 64-QAM modulation constellations [6].

A second video bitstream can also be multiplexed with the first one by the inner interleaver, when the DVB modem is in its so-called hierarchical mode [1]. The choice of modulation constellations in this mode is between 16-QAM and 64-QAM. We shall be employing this transmission mode, when the so-called data partitioning scheme, of Section V, is used to split the incoming MPEG-2 video bitstream into two video bit-protection classes with one class having a higher grade of protection or priority than the other one. The higher priority video bits will be mapped to the MSB's of the modulation constellation points and the lower priority video bits to the LSB's of the QAM-constellation [6]. For 16-QAM and 64-QAM, the two MSB's of each 4- or 6-bit QAM symbol will contain the more important video data. The lower priority video bits will then be mapped to the lower significance 2 bits and 4 bits of 16-QAM and 64-QAM, respectively [6].

These QPSK, 16-QAM, or 64-QAM symbols are then distributed over the OFDM carriers [6]. The parameters of the OFDM system are presented in Table II.

Beside implementing the standard DVB-T system as a benchmark, we have improved the system by replacing the convolutional coder by a turbo codec [17], [18]. The turbo codec's parameters used in our investigations are displayed in Table III. The block diagram of the turbo encoder is shown in Fig. 10. The turbo encoder is constructed of two component encoders. Each component encoder is a half-rate convolutional encoder, whose parameters are listed in Table III. The two component encoders are used to encode the same input bits, although the input bits of the second component encoder are interleaved before encoding. The output bits of the two component codes are punctured and multiplexed, in order to form a single output bitstream. The component encoder used here is known as a half-rate recursive systematic convolutional encoder (RSC) [19]. It generates one parity bit and one systematic output bit for every input bit. In order to provide an overall coding rate of $R=1 / 2$, half the output bits from the two encoders must be punctured. The puncturing arrangement used in our work is to transmit all the systematic bits from the first encoder and every other parity bit from both encoders [20]. We note here that one iteration of the turbo decoder involves two so-called Logarithmic Maximum A-Posteriori (LogMAP) [21] decoding operations, which we repeated for the 8 iterations. Hence, the total turbo decoding complexity is about 16 times higher than a constraint length $K=3$ constituent convolutional decoding. Therefore the turbo decoder exhibits a similar complexity to the $K=7$ convolutional decoder.

In this section, we have given an overview of the standard and enhanced DVB-T system, which we have used in our experiments. Readers interested in further details of the DVB-T system are referred to the DVB-T standard [1]. The performance of the standard DVB-T system and the turbo coded system is characterized in Sections VII and VIII for nonhierarchical and hierarchical transmissions, respectively. Let us now briefly consider the multipath channel model used in our investigations. 


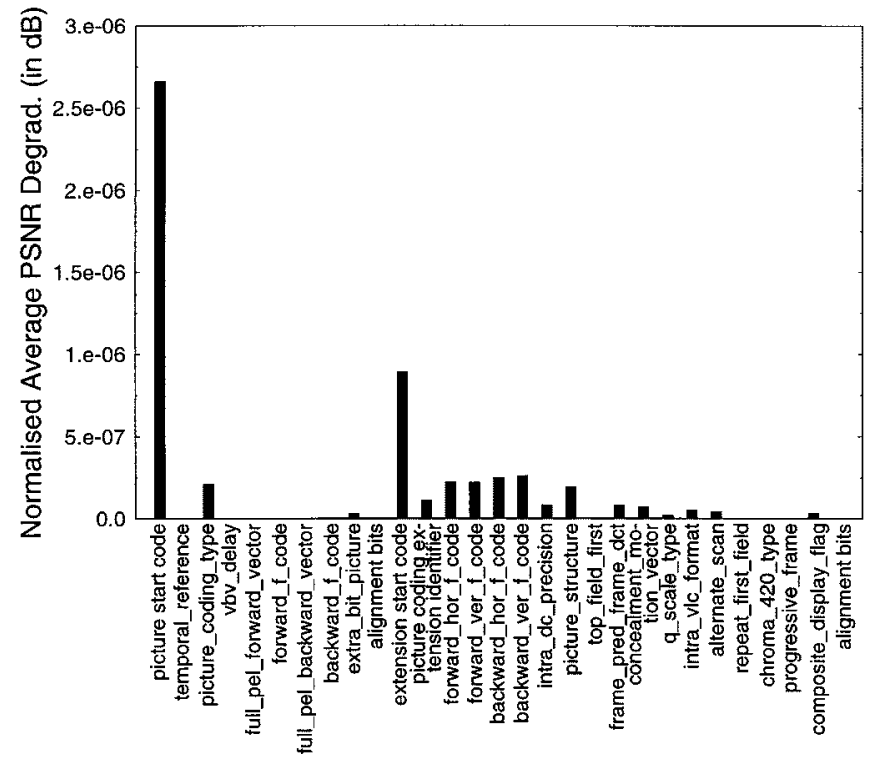

(a)

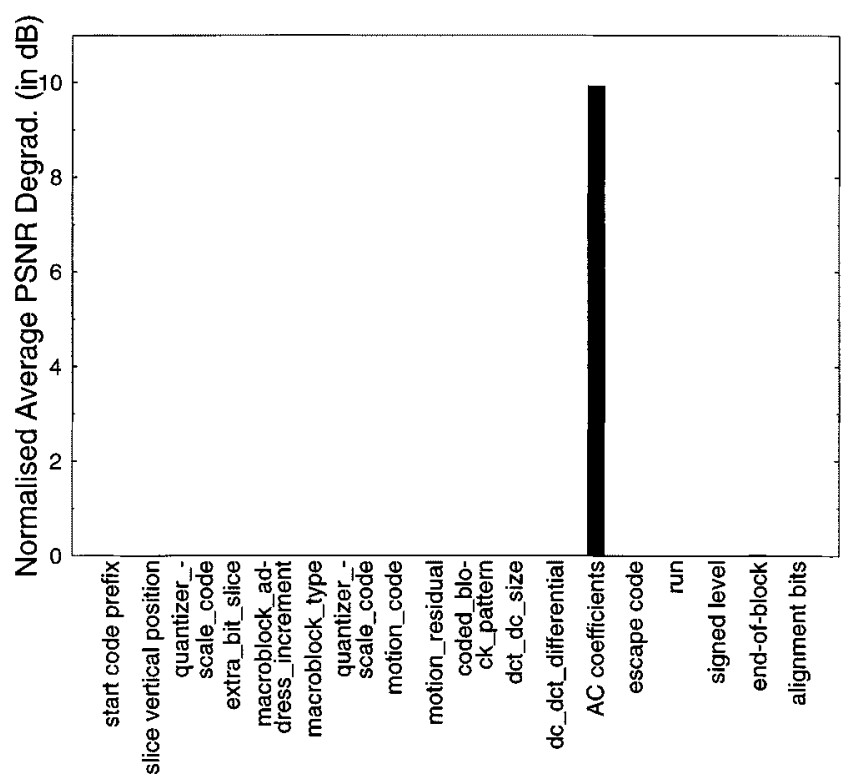

(b)

Fig. 7. Normalized average PSNR degradation for the various parameters in (a) Picture Header Information and Picture Coding Extension and (b) Slice-, Macroblock-, and Block-Layers, normalized to the probability of occurrence of the respective parameters in the bitstream and the probability of bits being allocated to the parameter for the "Miss America" QCIF video sequence encoded at $30 \mathrm{frame} / \mathrm{s}$ and $1.15 \mathrm{Mbit} / \mathrm{s}$.

\section{Channel Model}

The channel model employed in this study was the twelve-path COST 207 [22] hilly terrain (HT) type impulse response, with a maximal relative path delay of $19.9 \mu \mathrm{s}$. This channel was selected in order to provide a worst-case propagation scenario for the DVB-T system employed in our study.

In the system characterized here, we have used a carrier frequency of $500 \mathrm{MHz}$ and a sampling rate of 7/64 $\mu \mathrm{s}$. Each of the channel paths was faded independently obeying a Rayleigh fading distribution, according to a normalized Doppler frequency of $10^{-5}$ [4]. This corresponds to a worst-case vehicular velocity of about $200 \mathrm{~km} / \mathrm{h}$. The unfaded impulse response is

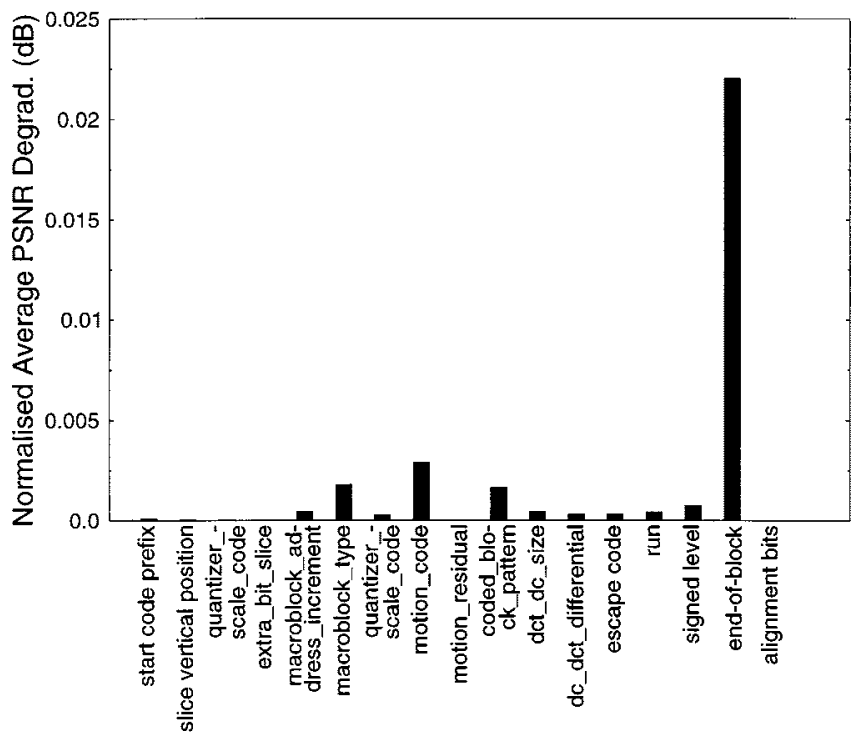

Fig. 8. This bar chart is the same as Fig. 7(b), although the normalized average PSNR degradation for the AC DCT coefficients was omitted in order to show the average PSNR degradation of the other parameters. This bar chart is presented for the "Miss America" QCIF video sequence encoded at 30 frame/s and 1.15 $\mathrm{Mbit} / \mathrm{s}$ case.

depicted in Fig. 11. For the sake of completeness we note that the standard COST 207 channel model was defined in order to facilitate the comparison of different GSM implementations [4] under identical conditions. The associated bit rate was 271 $\mathrm{kbit} / \mathrm{s}$, while in our investigations the bit rate of DVB-quality transmissions can be as high as $20 \mathrm{Mbit} / \mathrm{s}$, where there is a higher number of resolvable multipath components within the dispersion-range considered. However, the performance of various wireless transceivers is well understood by the research community over this standard COST 207 channel and hence its employment is beneficial in benchmarking terms. Furthermore, since the OFDM modem has 2048 subcarriers, the subcarrier signaling rate is effectively 2000-times lower than our maximum DVB-rate of $20 \mathrm{Mbit} / \mathrm{s}$, corresponding to 10 $\mathrm{kbit} / \mathrm{s}$. At this subchannel rate, the individual subchannel can be considered nearly frequency-flat. In summary, in conjunction with the $200 \mathrm{~km} / \mathrm{h}$ vehicular speed the investigated channel conditions constitute a pessimistic scenario.

In order to facilitate unequal error protection, the data partitioning procedure of the MPEG-2 video bitstream is considered next.

\section{DATA PARTITIONING SChEME}

Efficient bitstream partitioning schemes for H.263-coded video were proposed for example by Gharavi and Alamouti [23], which were evaluated in the context of the third-generation mobile radio standard proposal known as IMT-2000. As portrayed in Figs. 7 and 8, the corrupted variable-length coded DCT coefficients inflict a high video PSNR degradation. Assuming that all MPEG-2 header information is received correctly, the fidelity of the reconstructed images at the receiver is dependent on the number of correctly decoded DCT coefficients. However, the subjective effects of the loss of higher spatial frequency DCT coefficients are less dramatic compared 


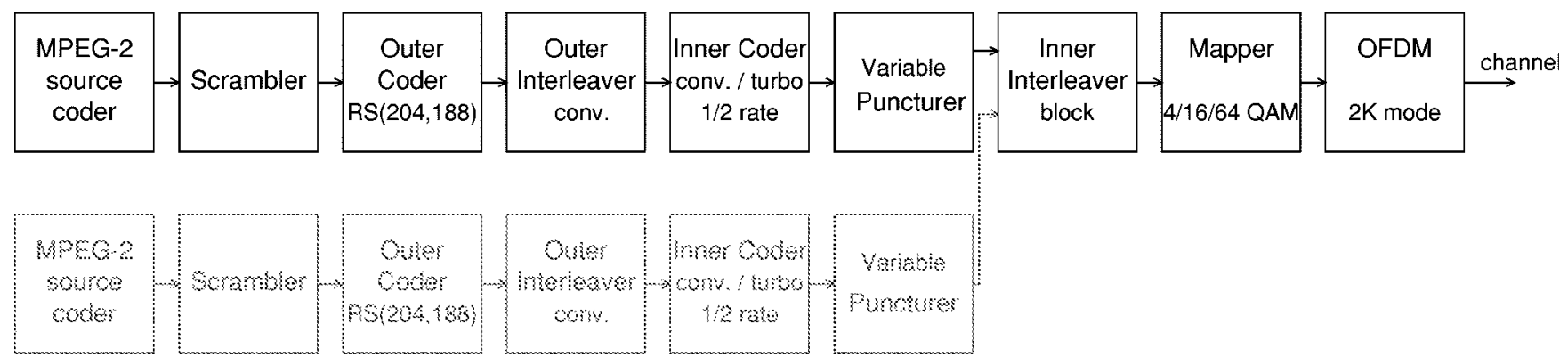

Fig. 9. Schematic of the DVB terrestrial transmitter functions.

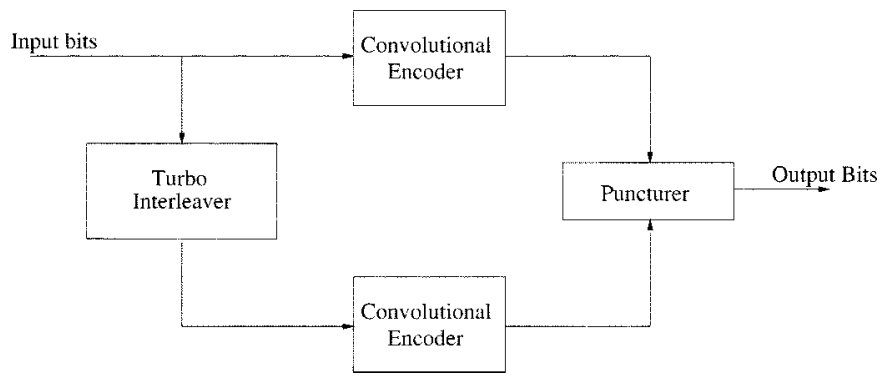

Fig. 10. Block diagram of turbo encoder.

TABLE I

PaRAMETERS OF the $C C(n, k, K)$ CONVOlutional INNER ENCODER OF THE DVB-T MODEM

\begin{tabular}{|l|c|}
\hline Convolutional Coder Parameters \\
\hline Code Rate & $1 / 2$ \\
Constraint Length & 7 \\
$n$ & 2 \\
$k$ & 1 \\
Generator Polynomials (octal format) & 171,133 \\
\hline
\end{tabular}

TABLE II

PARAMETERS OF THE OFDM MODULE USED IN THE DVB-T MODEM [1]

\begin{tabular}{|l|c|}
\hline OFDM Parameters \\
\hline Total number of subcarriers & $2048(2 \mathrm{~K}$ mode) \\
Number of effective subcarriers & 1705 \\
OFDM symbol duration $T_{s}$ & $224 \mu \mathrm{s}$ \\
Guard Interval & $T_{s} / 4=56 \mu \mathrm{s}$ \\
Total symbol duration & $280 \mu \mathrm{s}$ \\
(inc. Guard Interval) & $4464 \mathrm{~Hz}$ \\
Consecutive subcarrier spacing $1 / T_{s}$ & $7.61 \mathrm{MHz}$ \\
DVB channel spacing & $7 / 64 \mu \mathrm{s}$ \\
QPSK and QAM symbol period & \\
\hline
\end{tabular}

to that of the lower spatial frequency DCT coefficients. The splitting of the MPEG-2 video bitstream into two different integrity bitstreams is referred to as data partitioning [12]. Recall from Section III that the hierarchical 16- and 64-QAM DVB-T transmission scheme enables us to multiplex two unequally protected MPEG-2 video bitstreams for transmission. This section describes the details of the MPEG-2 data partitioning scheme [12].

Fig. 12 shows the block diagram of the data partitioning scheme, which splits an MPEG-2 video bitstream into two resultant bitstreams. The position at which the MPEG-2 bitstream is split is based on a variable referred to as the priority breakpoint (PBP) [12]. The PBP can be adjusted at the beginning of the encoding of every MPEG-2 image slice, based on the buffer "occupancy" or "fullness" of the two output buffers. For example, if the high-priority buffer is $80 \%$ full and the low-priority buffer is only $40 \%$ full, the rate control module would have to adjust the PBP such that more data is directed to the low-priority partition. This measure is taken to avoid high-priority buffer overflow and low-priority buffer underflow events. The valid values for the MPEG-2 PBP are summarized in Table IV [12].

There are two main stages in updating the PBP. The first stage involves the rate control module of Fig. 12 in order to decide on the preferred new PBP value for each partition based on its individual buffer occupancy and on the current value of the PBP. The second stage then combines the two desired PBP's based on the buffer occupancy of both buffers in order to produce a new PBP.

The updating of the PBP in the first stage of the rate control module is based on a heuristic approach, similar to that suggested by Aravind et al. [24]. The update procedure is detailed in Algorithm 1, which is discussed below and augmented by a numerical example at the end of this section.

The variable "sign" is used in Algorithm 1, in order to indicate how the PBP has to be adjusted in the high- and low-priority MPEG-2 partitions, so as to arrive at the required target buffer occupancy. More explicitly, the variable "sign" in Algorithm 1 is necessary, because the MPEG-2 PBP values [12] shown in Table IV indicate the amount of information which should be directed to the high-priority partition. Therefore, if the lowpriority partition requires more data, then the new PBP must be lower than the current PBP. By contrast, for the high-priority partition a higher PBP implies obtaining more data.

Once the desired PBP's for both partitions have been acquired with the aid of Algorithm 1, Algorithm 2 is invoked to compute the final PBP for the current MPEG-2 image slice. The inner working of these algorithms will be augmented by a numerical example at the end of this section. There are two main cases to consider in Algorithm 2. The first one, occurs when both partitions have a buffer occupancy of less than $50 \%$. By using the reciprocal of the buffer occupancy in Algorithm 2 as a weighting factor during the computation of the PBP adjustment value "delta," the algorithm will favor the new PBP decision of the less occupied buffer in order to fill the buffer with more data in the current image slice. This is simply because the buffer is closer to underflow and hence increasing the PBP according to its instructions will assist in preventing the particular 


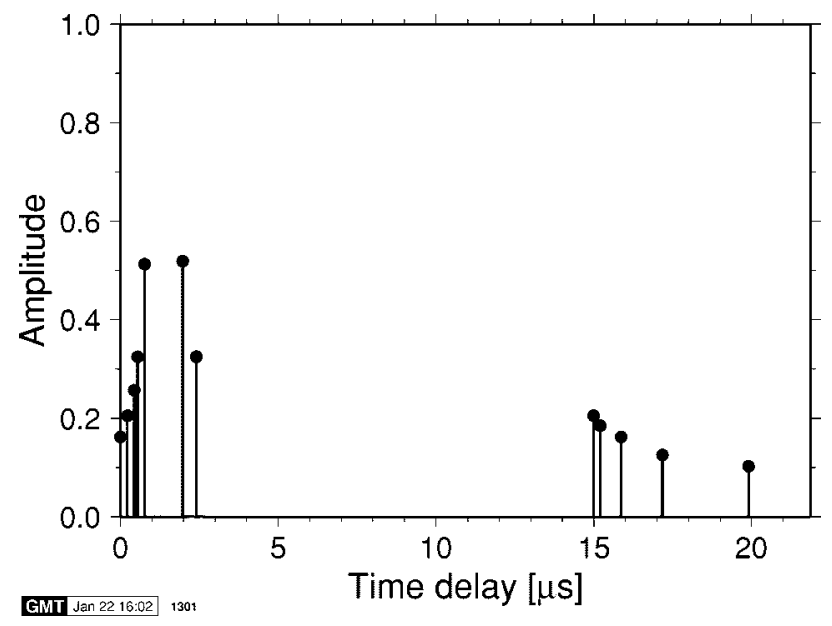

Fig. 11. COST 207 hilly terrain (HT) type impulse response.

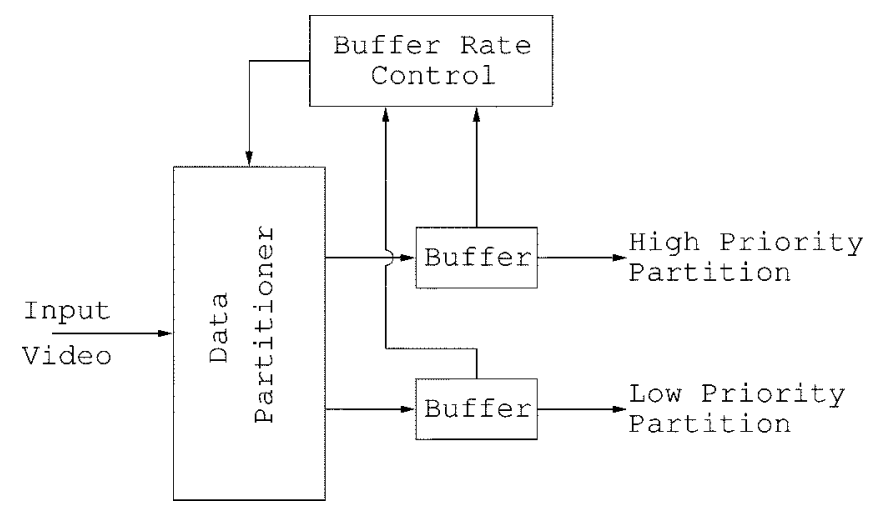

Fig. 12. Block diagram of the data partitioner and rate controller.

TABLE III

PARAMETERS OF THE INNER TURBo ENCODER USED TO REPLACE THE DVB-S SYSTEM'S $K=7$ CONVOLUTIONAL ENCODER OF Table I (RSC: RECURSIVE SySTEMATIC CODE)

\begin{tabular}{|l|c|}
\hline \multicolumn{2}{|l|}{ Turbo Coder Parameters } \\
\hline Turbo Code Rate & $1 / 2$ \\
Input Block Length & 17952 bits \\
Interleaver Type & Random \\
Number of Turbo Decoder Iterations & 8 \\
\hline \hline Turbo Encoder Component Code Parameters \\
\hline Component Code Encoder Type & Recursive \\
& Systematic \\
& Convolutional \\
Component Code Decoder Type & (RSC) \\
Constraint Length & Log-MAP $[21]$ \\
$n$ & 2 \\
$k$ & 1 \\
Generator Polynomials (octal format) & 7,5 \\
\hline
\end{tabular}

buffer from underflowing. On the other hand, when both buffers experience a buffer occupancy of more than $50 \%$, the buffer occupancy itself is used as a weighting factor instead. Now, the algorithm will instruct the buffer having a higher occupancy to have its desired PBP adjusted such that less data is inserted into it in the current MPEG-2 image slice. Hence, buffer overflow problems are alleviated with the aid of Algorithms 1 and 2.
The new PBP value is then compared to its legitimate range tabulated in Table IV. Furthermore, we restricted the minimum PBP value such that $I-, P$-, and $B$-pictures have minimum PBP values of 64,3 , and 2 , respectively. Since $B$-pictures are not used for future predictions, it was decided that their data need not be protected as strongly as that of the $I$ - and $P$-pictures. As for $P$-pictures, Ghanbari and Seferidis [25] showed that correctly decoded motion vectors alone can still provide a subjectively pleasing reconstruction of the image, even if the DCT coefficients were discarded. Hence, the minimum MPEG-2 bitstream splitting point or PBP for $P$-pictures has been set to be just before the coded block pattern parameter, which would then ensure that the motion vectors would be mapped to the high-priority partition. Upon receiving corrupted DCT coefficients they would be set to zero, which corresponds to setting the motion-compensated error residual of the macroblock concerned to zero. For $I$-pictures the fidelity of the reconstructed image is dependent on the number of DCT coefficients that can be decoded successfully. Therefore, the minimum MPEG-2 bitstream splitting point or PBP was set to include at least the first runlength coded DCT coefficient.

Below we demonstrate the operation of Algorithms 1 and 2 with the aid of a simple numerical example. We shall assume that the PBP prior to the update is 75 and the buffer occupancy for the high- and low-priority partition buffers is $40 \%$ and $10 \%$, respectively. Considering the high-priority partition, according to the buffer occupancy of $40 \%$ Algorithm 1 will set the desired PBP update difference denoted by "diff" for the PBP to +2 and this desired update is referred to as diff High Priority $_{\text {in Algo- }}$ rithm 2. For the low-priority partition, according to the buffer occupancy of 10\% Algorithm 1 will set the desired update for the PBP to -2 , since the sign of diff is changed by Algorithm 1. The desired PBP update for the low-priority partition is re-

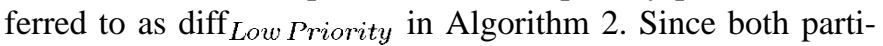
tion buffers' occupancy is less than 50\%, Algorithm 2 will use the reciprocal of the buffer occupancy as the weighting factor, which will then favor the desired update of the low-priority partition due to its $10 \%$ occupancy. The final update value-which is denoted by delta in Algorithm 2-is equal to -2 (after being rounded up). Hence, according to Step 2 of Algorithm 2 the new PBP is 73. This means that for the current MPEG-2 image slice more data will be directed into the low-priority partition, in order to prevent buffer underflow since PBP was reduced from 75 to 73 according to Table IV.

Apart from adjusting the PBP values from one MPEG-2 image slice to another to avoid buffer underflow or overflow, the output bitrate of each partition buffer must be adjusted such that the input bit rate of the inner interleaver and modulator in Fig. 9 is properly matched between the two partitions. Specifically, in the 16-QAM mode the two modem subchannels have an identical throughput of 2 bits per 4-bit symbol. By contrast, in the 64-QAM mode there are three 2-bit subchannels per 6-bit 64-QAM symbol, although the standard [1] recommends using a higher-priority 2-bit and a lower-priority 4-bit subchannels. Hence, it is imperative to take into account the redundancy added by forward error correction (FEC), especially when the two partition's FEC's operate at different code rates. Fig. 13 shows a block diagram of the DVB-T system operating in the 


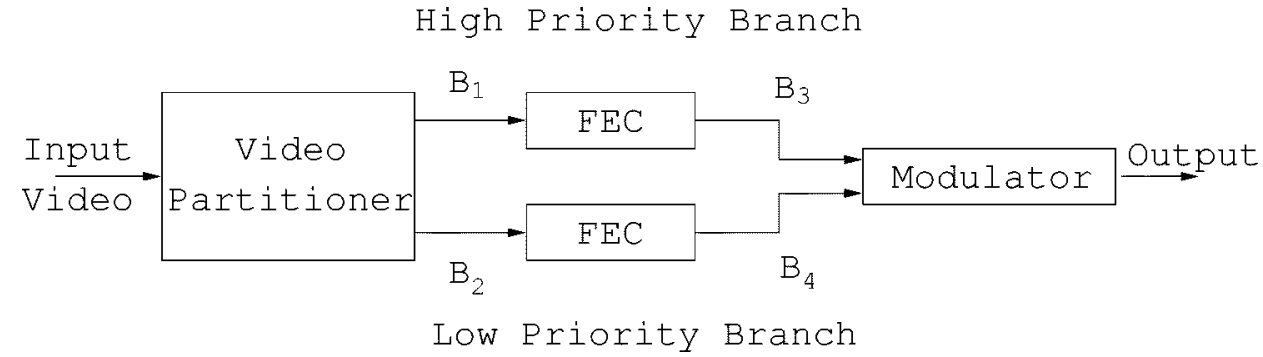

Fig. 13. Video partitioning scheme for the DVB-T system operating in hierarchical mode.

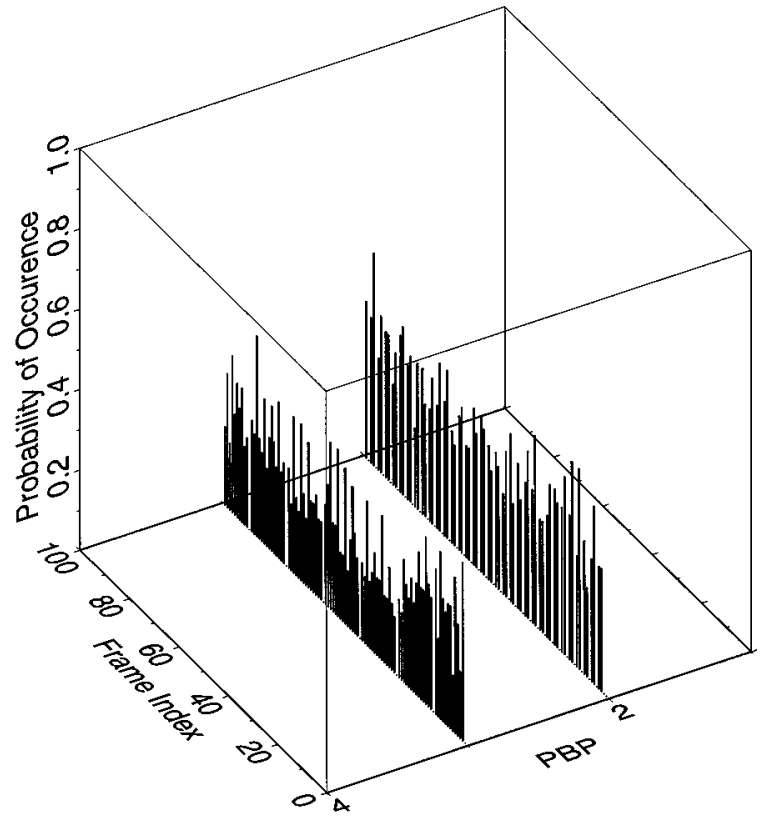

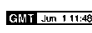

(a)

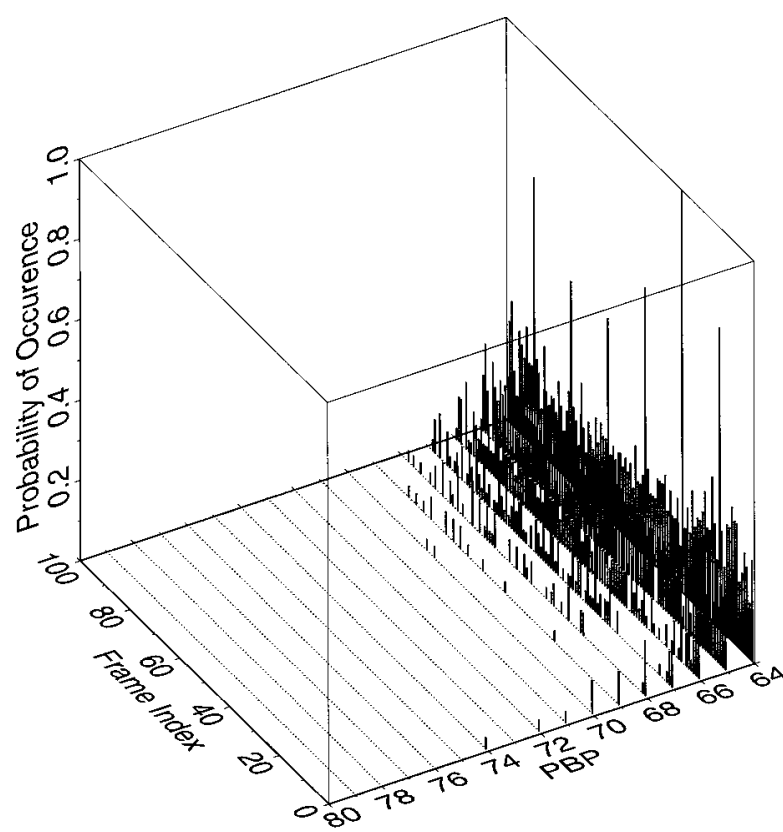

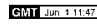

(b)

Fig. 14. Evolution of the probability of occurrence of PBP values from one picture to another of the $704 \times 576$-pixel "Football" video sequence for Scheme 1 of Table VI. hierarchical mode and receiving its input from the video partitioner. The FEC module represents the concatenated coding system, consisting of a Reed-Solomon codec [4] and a convolutional codec [4]. The modulator can invoke both 16-QAM and 64-QAM [6]. We shall now use an example to illustrate the choice of the various partitioning ratios summarized in Table V.

We shall assume that 64-QAM is selected and the high- and low-priority video partitions employ rate $1 / 2$ and 3/4 convolutional codes, respectively. This scenario is portrayed in the third line of the 64-QAM section of Table V. We do not have to take the Reed-Solomon code rate into account, since both partitions invoke the same Reed-Solomon codec. Based on these facts and upon referring to Fig. 13, the input bitrates $B_{3}$ and $B_{4}$ of the modulator must be in the ratio $1: 2$, since the two MSB's of the 64-QAM constellation are assigned to the high-priority video partition and the remaining four bits to the low-priority video partition.

At the same time, the ratio of $B_{3}$ to $B_{4}$ is related to the ratio of $B_{1}$ to $B_{2}$ with the FEC redundancy taken into account, requiring:

$$
\begin{aligned}
\frac{B_{3}}{B_{4}} & =\frac{2 \times B_{1}}{\frac{4}{3} \times B_{2}} \stackrel{64 \text { Q }}{=} \frac{1}{2} \\
& =\frac{3}{2} \cdot \frac{B_{1}}{B_{2}} \stackrel{64-\underline{Q} A M}{=} \frac{1}{2} \\
\frac{B_{1}}{B_{2}} & =\frac{1}{2} \times \frac{2}{3} \\
& =\frac{1}{3} .
\end{aligned}
$$

If, for example, the input video bitrate to the data partitioner module is $1 \mathrm{Mbit} / \mathrm{s}$, the output bitrate of the high- and low-priority partition would be $B_{1}=250 \mathrm{kbit} / \mathrm{s}$ and $B_{2}=750 \mathrm{kbit} / \mathrm{s}$, respectively, according to the ratio indicated by (2).

In this section, we have outlined the operation of the data partitioning scheme, which we used in the DVB-T hierarchical transmission scheme. Its performance in the context of the overall system will be characterized in Section VIII. Let us, however, first evaluate the BER-sensitivity of the partitioned MPEG-2 bitstream to randomly distributed bit errors using various partitioning ratios.

\section{Performance of Data the Partitioning Scheme}

Let us consider here the 16-QAM modem and refer to the equally split rate-1/2 convolutional coded high- and low-priority scenario as Scheme 1. Furthermore, the 16-QAM rate-1/3 


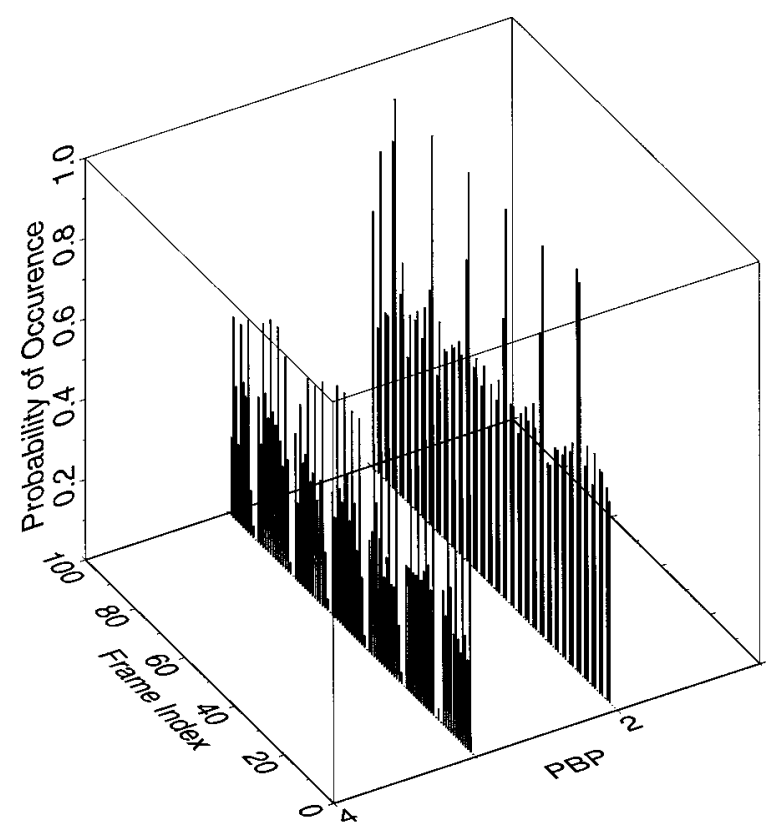

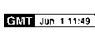

(a)

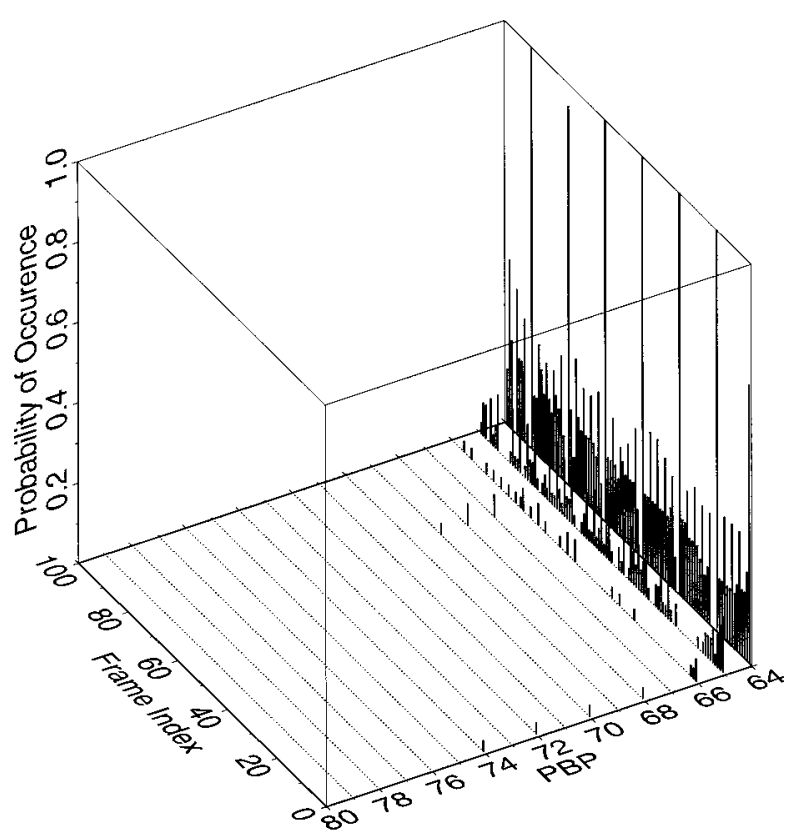

GQRT JUn 111:40

(b)

Fig. 15. Evolution of the probability of occurrence of PBP values from one picture to another of the $704 \times 576$-pixel "Football" video sequence for Scheme 2 of Table VI.

convolutional coded high-priority data and rate- $2 / 3$ convolutional coded low-priority data based scenario is referred to here as Scheme 2. Lastly, the 16-QAM rate-2/3 convolutional coded high-priority data and rate-1/3 coded low-priority data based partitioning scheme is termed as Scheme 3. We then programmed the partitioning scheme of Fig. 13 for maintaining the required splitting ratio $B_{1} / B_{2}$, as seen in Table VI. This was achieved by continuously adjusting the PBP using Algorithms 1 and 2. The $704 \times 576$-pixel "Football" High
Definition Television (HDTV) video sequence was used in these investigations.

Figs. 14-16 show the relative frequency at which a particular PBP value occurs for each image of the "Football" video sequence for the three different schemes of Table VI mentioned earlier. The reader may recall from Table IV that the PBP values indicate the proportion of encoded video parameters, which are to be directed into the high-priority partition. As the PBP value increases, the proportion of video data mapped to the high-priority partition increases and vice versa. Comparing Figs. 14-16, we observe that Scheme 3 has the most data in the high-priority partition associated with the high PBP's of Table IV, followed by Scheme 1 and Scheme 2. This observation can be explained as follows. We shall consider Scheme 3 first. In this scheme, the high-priority video bits are protected by a rate- $2 / 3$ convolutional code and mapped to the higher-integrity 16-QAM subchannel. By contrast, the low-priority video bits are encoded by a rate-1/3 convolutional code and mapped to the lower-integrity 16-QAM subchannel. Again, assuming that 16-QAM is used in our experiment according to line 3 of Table VI, 2/3 of the video bits will be placed in the high-priority 16-QAM partition and the remaining video bits in the low-priority 16-QAM partition, following the approach of (2). The BER difference of the 16-QAM subchannels depend on the channel error statistics, but the associated BER's are about a factor of 2-3 different [6]. In contrast to Scheme 3, Scheme 2 will have $1 / 3$ of the video bits placed in the high-priority 16-QAM partition and the remaining 2/3 of the video bits mapped to the low-priority 16-QAM partition, according to line 2 of Table VI. Lastly, Scheme 1 will have half of the video bits in the high- and low-priority 16-QAM partitions, according to line 1 of Table VI. This explains our observation in the context of Scheme 3 in Fig. 16, where a PBP value as high as 80 is achieved in some image frames. However, each PBP value encountered has a lower probability of being selected, since the total number of 3600 occurrences associated with investigated 3600 MPEG-2 video slices per 100 image frames is spread over a higher variety of PBP's. Hence, Scheme 3 directs about 2/3 of the original video bits after 2/3-rate coding to the high-priority 16-QAM subchannel. This observation is in contrast to Scheme 2 of Fig. 15, where the majority of the PBP's selected are only up to the value of 65 . This indicates that about $2 / 3$ of the video bits are concentrated in the lower-priority partition, as indicated in line 2 of Table VI.

Figs. 17(a) to 19(a) show the average probability at which a particular PBP value is selected by the rate control scheme, as discussed in Section V, during the encoding of the video sequence. Again, we observe that Scheme 3 encounters the widest range of PBP values, followed by Scheme 1 and Scheme 2, respectively, since according to Table VI these schemes map a decreasing number of bits to the high-priority partition in this order.

We then embarked on quantifying the error sensitivity of the partitioning Schemes 1 to 3 characterized in Table VI, when each partition was subjected to randomly distributed bit errors, although in practice the error distribution will depend on the fading channel's characteristics. Specifically, the previously defined average PSNR degradation was evaluated for given error probabilities inflicting random errors imposed on one of the par- 


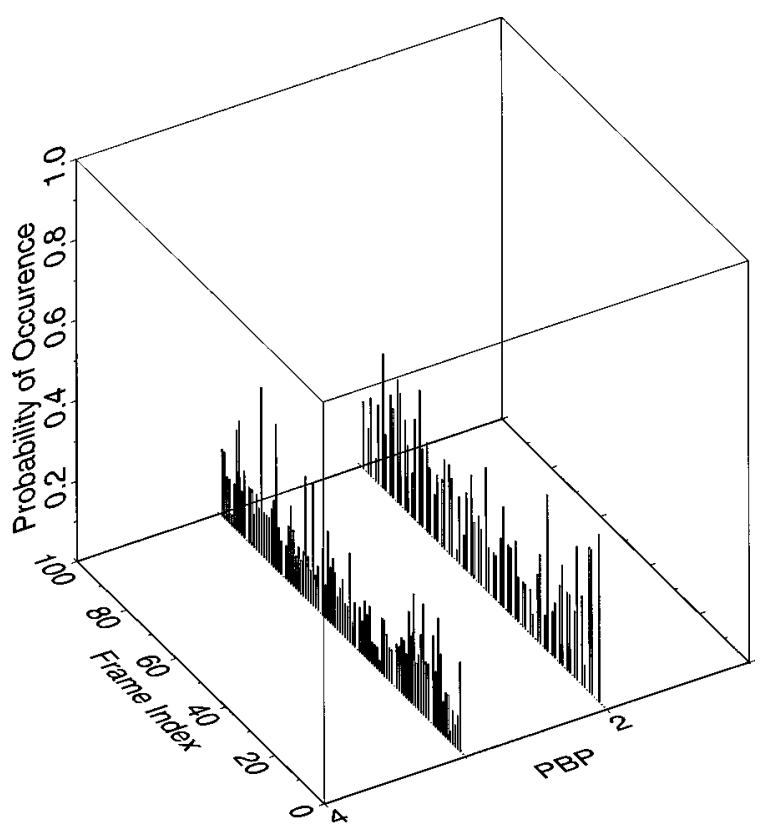

GhHT Un IIIIS0

(a)

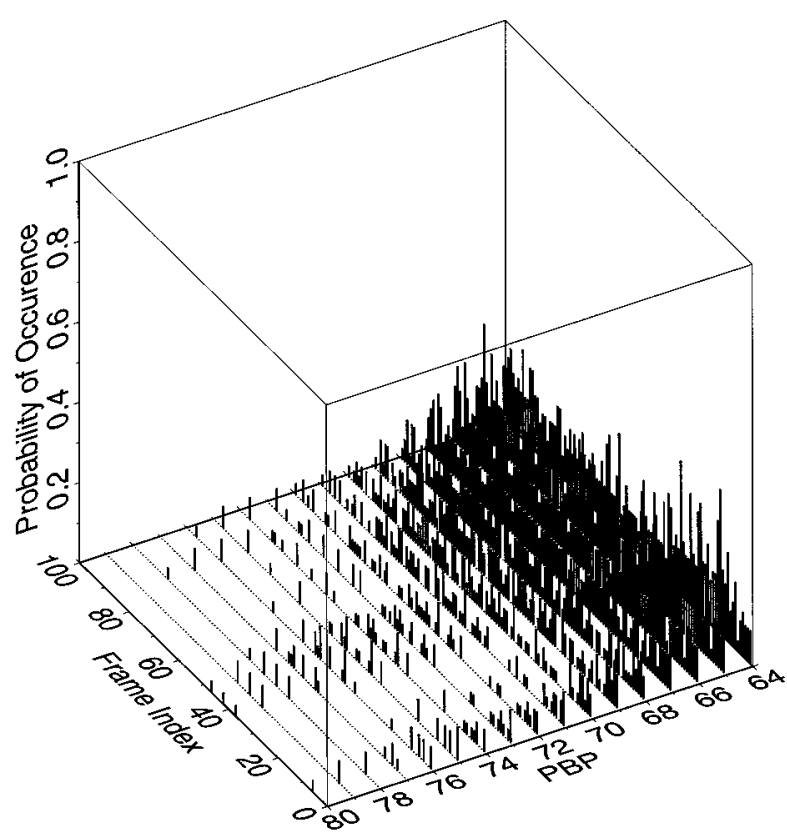

GMTI Uun 111.50

(b)

Fig. 16. Evolution of the probability of occurrence of PBP values from one picture to another of the $704 \times 576$-pixel "Football" video sequence for Scheme 3 of Table VI.

titions, while keeping the other partition error-free. These results are portrayed in Figs. 17(b), 18(b), and 19(b), for Schemes 1 to 3 , respectively.

Comparing Figs. 17(b) to 19(b), we observe that the average PSNR degradation exhibited by the three schemes of Table VI, when only their high-priority partitions are corrupted, is similar. The variations in the average PSNR degradation in these cases are caused by the different quantity of sensitive video bits, which resides in the high-priority partition. If we compare the performance of the schemes summarized in Table VI at a BER of
$2 \times 10^{-3}$, Scheme 3 experienced approximately $8.8 \mathrm{~dB}$ average video PSNR degradation, while Schemes 1 and 2 exhibited approximately $5 \mathrm{~dB}$ degradation. This trend was expected, since Scheme 3 had the highest portion of the video bits-namely 2/3-residing in the high-priority partition, followed by Scheme 1 hosting $1 / 2$ and Scheme 2 having 1/3 of the bits in this partition.

On the other hand, we can observe a significant difference in the average PSNR degradation measured for Schemes 1 to 3 of Table VI, when only the low-priority partitions are corrupted by comparing the curves shown as broken lines in Figs. 17(b) to 19 (b). Under this condition, Scheme 2 experienced approximately $16 \mathrm{~dB}$ average video PSNR degradation at a BER of $2 \times 10^{-3}$. By contrast, Scheme 1 exhibited an approximately 4 $\mathrm{dB}$ average video PSNR degradation, while Scheme 3 experienced about $7.5 \mathrm{~dB}$ degradation at this BER. The scheme with the highest portion of video bits in the lower-priority partition, i.e., Scheme 2, experienced the highest average video PSNR degradation. This observation correlates well with our earlier findings in the context of the high-priority partition scenario, where the partition holding the highest portion of the video bits in the error-impaired partition, exhibited the highest average PSNR degradation.

Having discussed our observations for the three schemes of Table VI from the perspective of the relative amount of video bits in one partition compared to the other, we shall now examine the details of the data partitioning process further, in order to relate them to our observations. Fig. 20 shows a typical example of an MPEG-2 video bitstream both prior to data partitioning and after data partitioning. There are two scenarios to be considered here, namely intra-frame coded macroblock partitioning and inter-frame coded macroblock partitioning. We have selected the PBP value of 64 from Table IV for the intraframe coded macroblock scenario and the PBP value of 3 for the inter-frame coded macroblock scenario, since these values have been selected frequently by the rate control arrangement for Schemes 1 and 2. This is evident from Figs. 14 and 15 as well as from Figs. 17(a) and 18(a). This implies, with the aid of Table VI and Fig. 20, that only the macroblock (MB) header information and a few low-frequency DCT coefficients will reside in the high-priority partition, while the rest of the DCT coefficients will be stored in the low-priority partition. These can be termed as base-layer and enhancement-layer, as seen in Fig. 20. In the worst-case scenario, where the entire enhancement-layer or low-priority partition data is lost due to a transmission error near the beginning of the associated low-priority bitstream, the MPEG-2 video decoder will only have the bits of the high-priority partition in order to reconstruct the encoded video sequence. Hence, the MPEG-2 decoder certainly cannot reconstruct good quality images. Although the results reported by Ghanbari and Seferidis [25] suggested that adequate video reconstruction is possible, provided that the motion vectors are correctly decoded, this observation is only true if the previous intra-coded frame is correctly reconstructed. If the previous intra-coded frame contains artifacts, these artifacts will be further propagated to forthcoming video frames by the motion vectors. By attempting to provide higher protection for the high-priority partition or base-layer, we have indirectly forced 
TABLE IV

Priority BreakPoint Values and The Associated MPEG-2 Parameters that Will Be Directed to the High-PRioRity PaRtition [12]. A Higher PBP Directs More Parameters to the High-Priority Partition. By CONTRAST, FOR the LOW-Priority Partition a Higher PBP IMPLIES OBTAINING LESS DATA

\begin{tabular}{|l|l|}
\hline PBP & Syntax elements in high-priority partition \\
\hline \hline 0 & Low-priority partition always has its PBP set to 0. \\
\hline 1 & Sequence, GOP, Picture and Slice layer information upto extra bit slice. \\
\hline 2 & Same as above and upto macroblock address increment. \\
\hline 3 & $\begin{array}{l}\text { Same as above plus including macroblock syntax elements, but excluding } \\
\text { coded block pattern. }\end{array}$ \\
\hline $4 \ldots 63$ & Reserved for future use. \\
\hline 64 & $\begin{array}{l}\text { Same as above plus including DC DCT coefficient and the first runlength } \\
\text { coded DCT coefficient. }\end{array}$ \\
\hline 65 & Same as above and up to the second runlength coded DCT coefficient. \\
\hline $64+x$ & Same as above and up to x runlength coded DCT coefficient. \\
\hline 127 & Same as above and up to 64 runlength coded DCT coefficient. \\
\hline
\end{tabular}

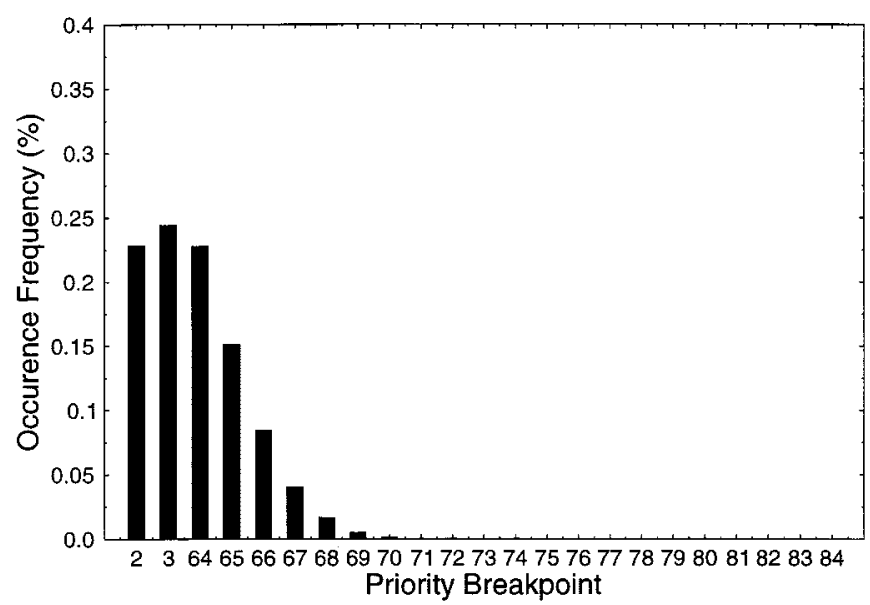

(a)

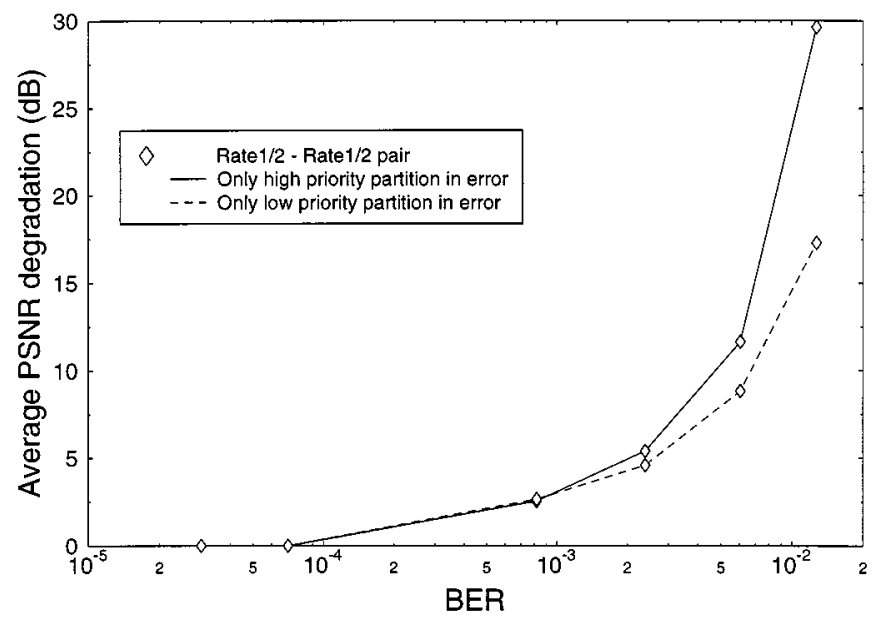

(b)

Fig. 17. (a) Histogram of the probability of occurrence for various priority breakpoints and (b) average PSNR degradation versus BER for rate-1/2 convolutional coded high- and low-priority data in Scheme 1 of Table VI.

the rate control scheme, of Section V, to reduce the proportion of video bits directed into the high-priority partition under the constraint of a given fixed bit rate, which is imposed by the 16-QAM subchannels.

In order to elaborate a little further, at a BER of $2 \times 10^{-3}$ Scheme 1 in Fig. 17(a) exhibited a near-identical PSNR degra-

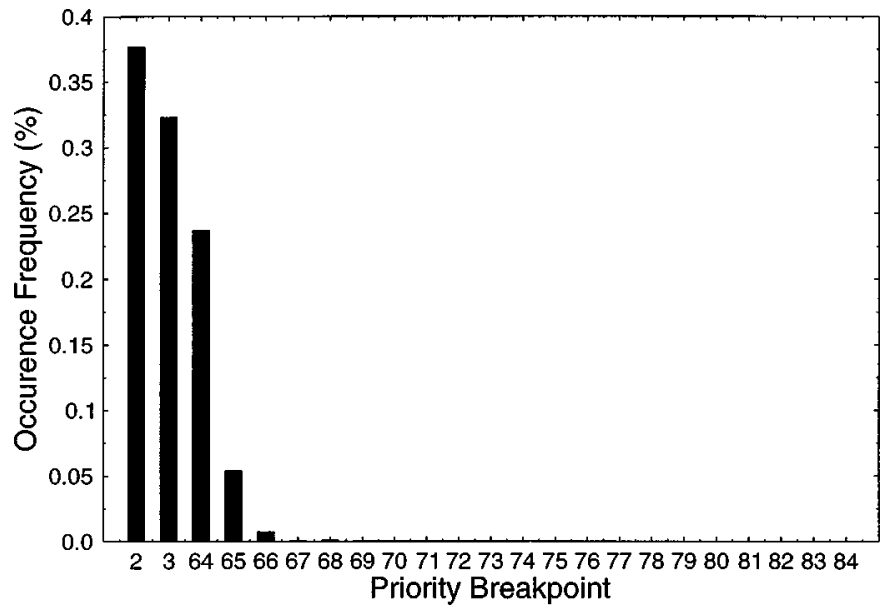

(a)

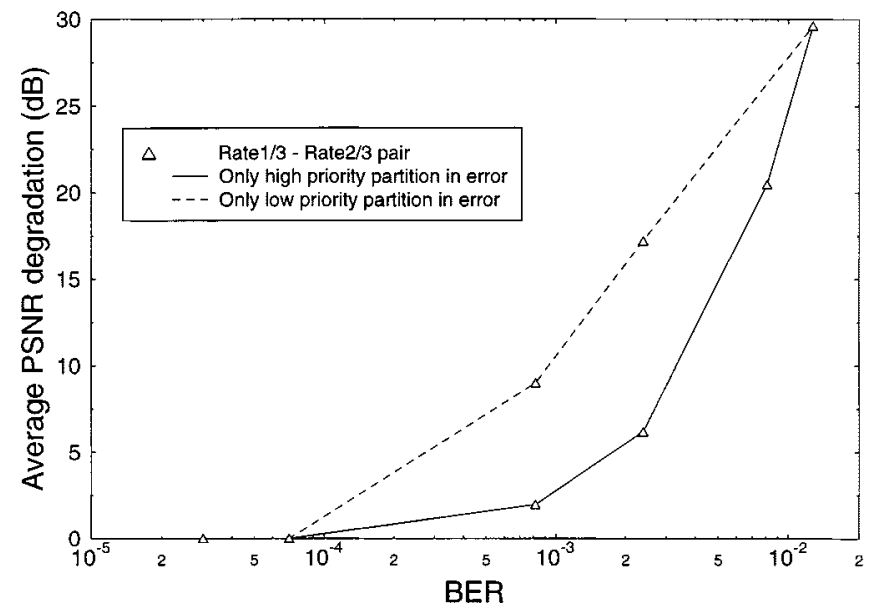

(b)

Fig. 18. (a) Histogram of the probability of occurrence for various priority breakpoints and (b) average PSNR degradation versus BER for the rate-1/3 convolutional coded high-priority data and rate $2 / 3$ convolutional coded low-priority data in Scheme2 of Table VI.

dation for the high- and low-priority video bits. Upon assigning more bits to the low-priority partition, in order to be able to accommodate a stronger FEC code in the high-priority partition results in an increased proportion of error-impaired bits and the associated higher error sensitivity seen in Fig. 18(b). As such, 
TABLE V

The Partitioning Ratios for the High- and Low-Priority Partition's Output Bitrate Based on the Modulation Mode and Code Rates Selected FOR THE DVB-T HIERARCHICAL MODE. THE LINE IN BOLD CORRESPONDS TO OUR WORKED EXAMPLE.

\begin{tabular}{|c|c|c|c|}
\hline Modulation & $\begin{array}{c}\text { Conv. Code Rate } \\
\text { (High Priority) }\end{array}$ & $\begin{array}{c}\text { Conv. Code Rate } \\
\text { (Low Priority) }\end{array}$ & $\begin{array}{c}\text { Partition Bit Rate Ratio } \\
\text { (High Priority - B1 : Low Priority - B2) }\end{array}$ \\
\hline \hline 16-QAM & $1 / 2$ & $1 / 2$ & $1: 1$ \\
& $1 / 2$ & $2 / 3$ & $3: 4$ \\
& $1 / 2$ & $3 / 4$ & $2: 3$ \\
& $1 / 2$ & $5 / 6$ & $3: 5$ \\
& $1 / 2$ & $7 / 8$ & $4: 7$ \\
& $2 / 3$ & $1 / 2$ & $4: 3$ \\
\hline 64-QAM & $1 / 2$ & $1 / 2$ & $1: 2$ \\
& $1 / 2$ & $2 / 3$ & $\mathbf{3}: 3$ \\
& $1 / 2$ & $3 / 4$ & $3: 10$ \\
& $1 / 2$ & $5 / 6$ & $2: 7$ \\
& $1 / 2$ & $7 / 8$ & $2: 3$ \\
\hline
\end{tabular}

TABLE VI

Summary of the Three Schemes Employed in Our Investigations into the Performance of the Data Partitioning Scheme. The FEC-Coded High-Priority Video Bitstream B3, as Shown in Fig. 3, WAS MapPed to the High-PrioRity 16-QAM Subchannel, WhiLE the LOW-PRIORITY B4-STREAM TO THE LOW-PRIORITY 16-QAM SUBCHANNEL

\begin{tabular}{|l|c|c|c|c|}
\hline & Modulation & $\begin{array}{c}\text { Conv. Code Rate } \\
\text { (High Priority - B1) }\end{array}$ & $\begin{array}{c}\text { Conv. Code Rate } \\
\text { (Low Priority - B2) }\end{array}$ & $\begin{array}{c}\text { Bit Rate Ratio } \\
\text { (High Priority - B1 : Low Priority - B2) }\end{array}$ \\
\hline \hline Scheme 1 & 16-QAM & $1 / 2$ & $1 / 2$ & $1: 1$ \\
Scheme 2 & 16-QAM & $1 / 3$ & $2 / 3$ & $1: 2$ \\
Scheme 3 & 16-QAM & $2 / 3$ & $1 / 3$ & $2: 1$ \\
\hline
\end{tabular}

TABLE VII

Summary of the Non-Hierarchical Performance Results Over NON-DiSPERSIVE AWGN CHANNELS TOLERATING A PSNR DEGRADATION OF 2 dB. THE BER MEASURE REFERS TO BER AFTER VITERBI OR TURBo DECODING.

\begin{tabular}{|l|l|r|r|r|}
\hline Mod. & Code & $\begin{array}{r}\text { CSNR } \\
(\mathrm{dB})\end{array}$ & $\tilde{E}_{\boldsymbol{b}} / N_{0}$ & BER \\
\hline QPSK & Turbo (1/2) & 1.02 & 1.02 & $6 \cdot 10^{-6}$ \\
\hline 64QAM & Turbo (1/2) & 9.94 & 5.17 & $2 \cdot 10^{-3}$ \\
\hline QPSK & Conv $(1 / 2)$ & 2.16 & 2.16 & $1.1 \cdot 10^{-3}$ \\
\hline 64QAM & Conv $(1 / 2)$ & 12.84 & 8.07 & $6 \cdot 10^{-4}$ \\
\hline QPSK & Conv $(7 / 8)$ & 6.99 & 4.56 & $2 \cdot 10^{-4}$ \\
\hline 64QAM & Conv $(7 / 8)$ & 19.43 & 12.23 & $3 \cdot 10^{-4}$ \\
\hline
\end{tabular}

there is a trade-off between the amount of video data protected and the code rate of the channel codec. As a comparison to the above scenarios in the context of Schemes 1 and 2, we shall now examine Scheme 3. In this scheme, more video data-namely half the bits-can be directed into the high-priority partition, as demonstrated by Fig. 16 due to encountering higher PBP's. This can also be confirmed with reference to Figs. 18(b) and 19(b) by observing the PSNR degradations associated with the curves plotted in broken lines. If the low-priority partition is lost in Scheme 3, its effect on the quality of the reconstructed images is less detrimental than that of Scheme 2, since Scheme 3 loses only half the bits, rather than $2 / 3$. Hence, it is interesting to note that Scheme 3 experiences slightly higher average PSNR degradation than Scheme 1 at a BER of $2 \times 10^{-3}$, when only the low-priority partition is lost in both cases, despite directing only $1 / 3$ rather than $1 / 2$ of the bits to the low-priority partition. This observation can be explained as follows.

Apart from partitioning the macroblock header information and the variable-length coded DCT coefficients into the highand low-priority partitions, synchronization information such as the Picture Header Information [12] is replicated in the enhancement layer, as suggested by Gharavi et al. [23] as well as the MPEG-2 standard [12]. The purpose is to enable the MPEG-2 decoder to keep the base- and enhancement-layers synchronized during decoding. An example of this arrangement is shown in Fig. 21. This resynchronization measure is only effective, when the picture start code of both the high- and low-priority partitions are received correctly. If the picture start code in the low-priority partition is corrupted, for example, the MPEG-2 decoder may not detect this PSC and all the data corresponding to the current image frame in the low-priority partition will be lost. The MPEG-2 decoder will then interpret the bits received for the low-priority partition of the next frame as the low-priority data expected for the current frame. As expected, due to this synchronization problem the decoded video would have a higher average PSNR degradation, than for the case where picture start codes are unimpaired. This explains our observation of a higher average PSNR degradation for Scheme 3, when only its lower-priority partition was corrupted by the transmission channel. On the other hand, in this specific experiment, Scheme 1 did not experience the loss of synchronization due to corruption of its picture start code. Viewing events from another perspective, by opting for allocating less useful video bits to the low-priority partition, the probability of transmission errors af- 


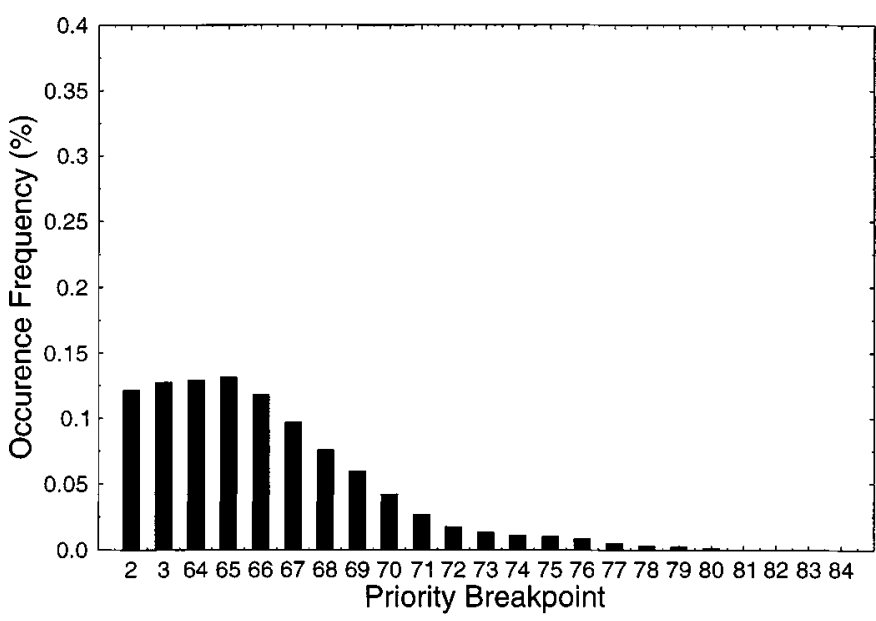

(a)

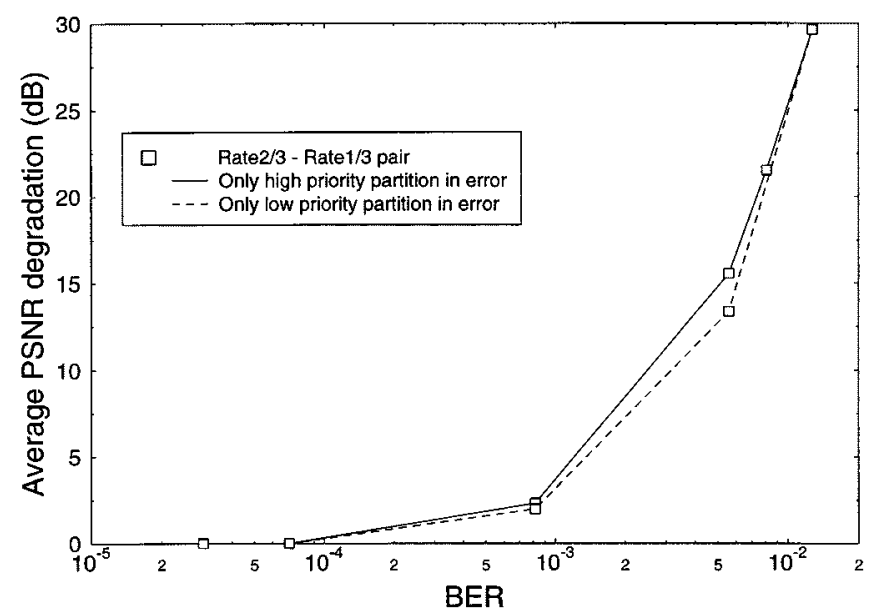

(b)

Fig. 19. (a) Histogram of the probability of occurrence for various priority breakpoints and (b) average PSNR degradation versus BER for the rate-2/3 convolutional coded high-priority data and rate-1/3 convolutional coded low-priority data in Scheme 3 of Table VI.

fecting the fixed-length PSC within the reduced-sized low-priority partition becomes higher.

These findings will assist us in explaining our observations in the context of the hierarchical transmission scheme of Section VIII, suggesting that the data partitioning scheme did not provide overall gain in terms of error resilience over the nonpartitioned case. Let us, however, consider first the performance of the nonhierarchical DVB-T scheme in Section VII.

\section{ViI. Performance of the DVB Terrestrial Scheme EMPLOYING NON-HIERARCHICAL TRANSMISSION}

In this section we shall elaborate on our findings when the convolutional code used in the standard nonhierarchical DVB scheme [1] is replaced by a turbo code. We will invoke a range of standard-compliant schemes as benchmarks. The $704 \times 576$-pixel HDTV-resolution "Football" video sequence was used in our experiments. The MPEG-2 decoder employs a simple error concealment algorithm to fill in missing portions of the reconstructed image in the event of decoding errors. The concealment algorithm will select the specific portion of the previous reconstructed image, which corresponds to the

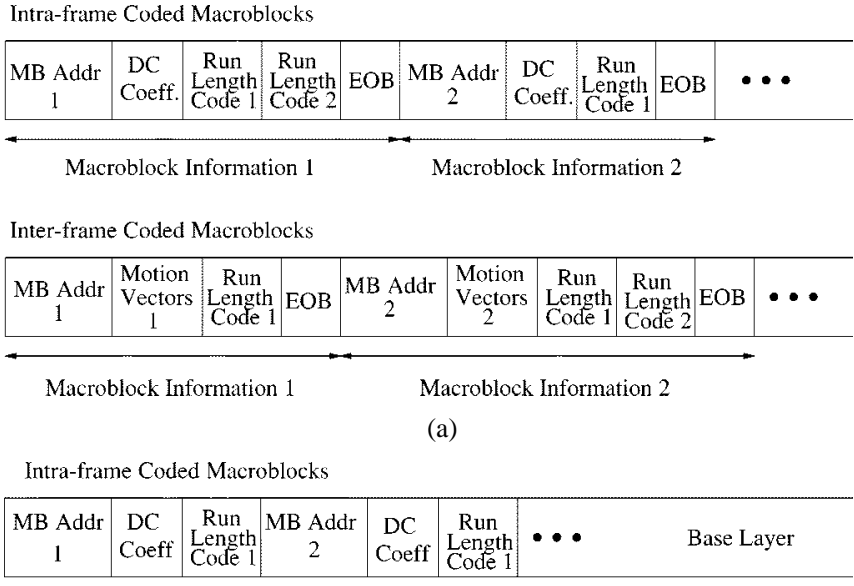

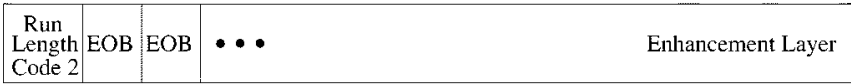

\begin{tabular}{|c|c|c|c|c|c|}
\hline $\begin{array}{c}\text { MB Addr } \\
1\end{array}$ & $\begin{array}{l}\text { Motion } \\
\text { Vectors } \\
1\end{array}$ & $\begin{array}{c}\text { MB Addr } \\
2\end{array}$ & $\begin{array}{c}\text { Motion } \\
\text { Vectors } \\
2\end{array}$ & $\bullet \bullet$ & Base Layer \\
\hline
\end{tabular}

\begin{tabular}{|c|c|c|c|c|c|c|}
\hline $\begin{array}{l}\text { Run } \\
\text { Length } \\
\text { Code } 1\end{array}$ & EOB & $\begin{array}{l}\text { Run } \\
\text { Length } \\
\text { Code } 1\end{array}$ & $\begin{array}{l}\text { Run } \\
\text { Length } \\
\text { Code } 2\end{array}$ & EOB & $\bullet \bullet$ & Enhancement Layer \\
\hline
\end{tabular}

(b)

Fig. 20. Example of video bitstream (a) before data partitioning and (b) after data partitioning for intra-frame coded macroblocks (MB) assuming a PBP of 64 and for inter-frame coded macroblocks assuming a PBP of 3.

\begin{tabular}{l} 
Base Layer (High Priority Partition) \\
\begin{tabular}{|c|c|c|c|c|c|c|c|c}
\hline $\begin{array}{c}\text { Picture } \\
\text { Header } \\
1\end{array}$ & $\begin{array}{c}\text { Slice } \\
\text { Header }\end{array}$ & $\begin{array}{c}\text { MB } \\
\text { Data }\end{array}$ & $\begin{array}{c}\text { Slice } \\
2\end{array}$ & $\begin{array}{c}\text { MB } \\
\text { Data }\end{array}$ & $\begin{array}{c}\text { Picture } \\
\text { Header }\end{array}$ & $\begin{array}{c}\text { Slice } \\
\text { Header } \\
1\end{array}$ & $\begin{array}{c}\text { MB } \\
\text { Data }\end{array}$ & $\ldots$ \\
\hline
\end{tabular} \\
\hline
\end{tabular}

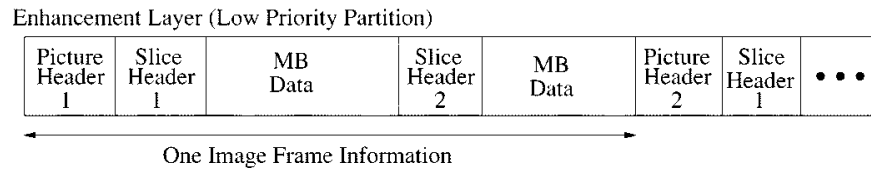

Fig. 21. Example of high level bitstream syntax structure of a data partitioned MPEG-2 video bitstream. The "MB data" shown in the diagram refers to the macroblock header information and to the variable length coded DCT coefficients, which have been partitioned as shown in Fig. 20.

missing portion of the current image, in order to conceal the errors.

In Fig. 22(a) and (b) and the bit error rate (BER) performance of the various modem modes in conjunction with our diverse channel coding schemes are portrayed over stationary, narrowband Additive White Gaussian Noise channels (AWGN), where the turbo codec exhibits a significantly steeper BER reduction in comparison to the convolutionally coded arrangements.

Specifically, comparing the performance of the various turbo and convolutional codes for QPSK and 64-QAM at a BER of $10^{-4}$, the turbo code exhibited an additional coding gain of about 2.24 and $3.7 \mathrm{~dB}$ respectively, when using half-rate codes in Fig. 22(a) and (b). Hence, the Peak Signal to Noise Ratio 


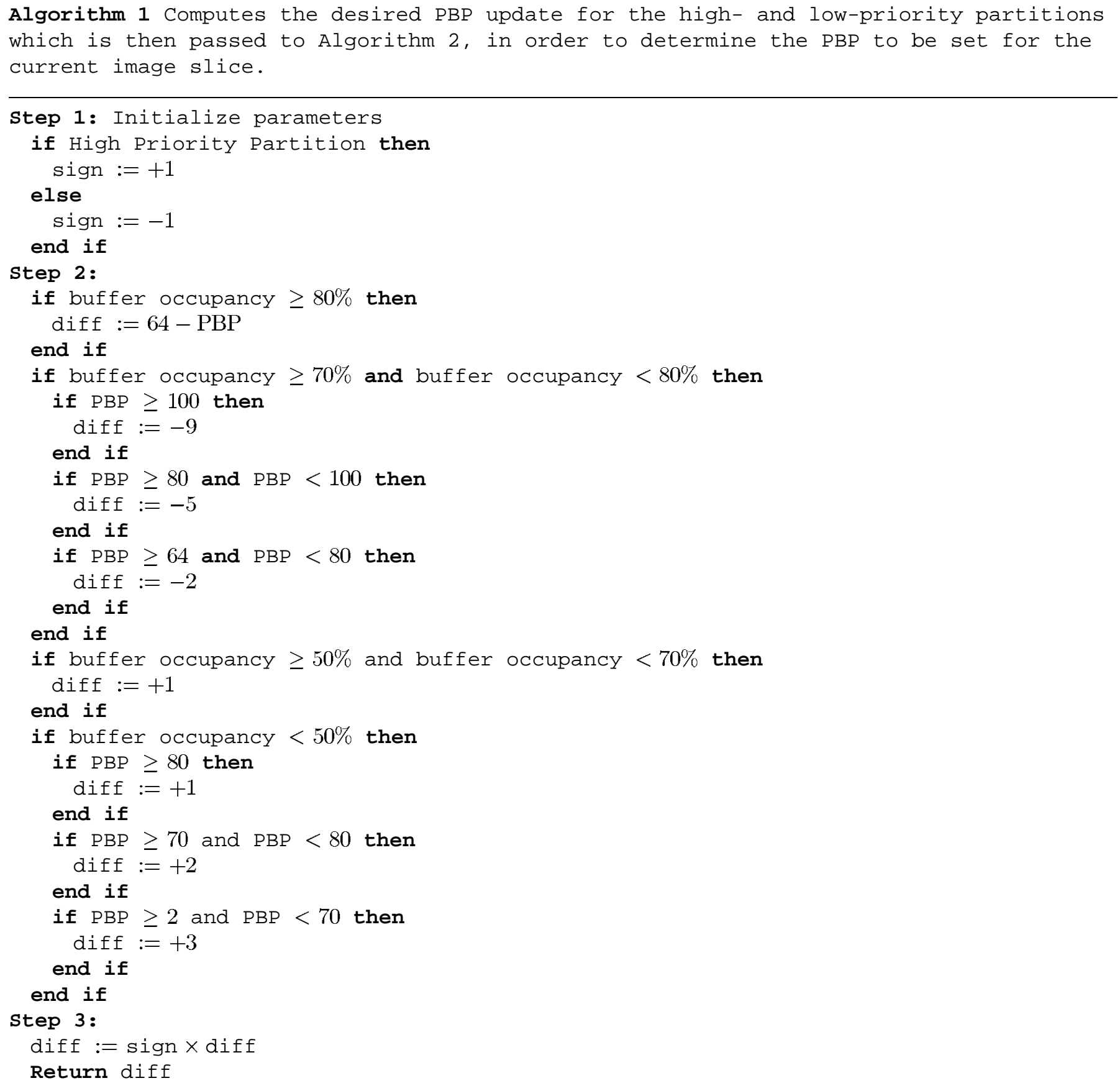

(PSNR) versus channel Signal to Noise Ratio (SNR) graphs in Fig. 25 demonstrate that approximately 2 and $3.5 \mathrm{~dB}$ lower channel SNR's are required in conjunction with the rate $1 / 2$ turbo codec for QPSK and 64-QAM, respectively, than for convolutional coding, in order to maintain high reconstructed video quality. The term unimpaired as used in Fig. 25 and Fig. 26 refers to the condition, where the PSNR of the MPEG-2 decoder's reconstructed image at the receiver is the same as the PSNR of the same image generated by the local decoder of the MPEG-2 video encoder, corresponding to the absence of channel—but not MPEG-2 coding-impairments.
Comparing the BER performance of the 1/2-rate convolutional decoder in Fig. 23(a) and the so-called Log-Map turbo decoder using eight iterations in Fig. 23(b) for QPSK modulation over the worst-case fading mobile channel of Fig. 11, we observe that at a BER of about $10^{-4}$ the turbo code provided an additional coding gain of $6 \mathrm{~dB}$ in comparison to the convolutional code. By contrast, for 64QAM using similar codes, a 5 $\mathrm{dB}$ coding gain was observed at this BER.

Similar observations were also made with respect to the average Peak Signal to Noise Ratio (PSNR) versus channel Signal to Noise Ratio (SNR) plots of Fig. 26. For example, for the 


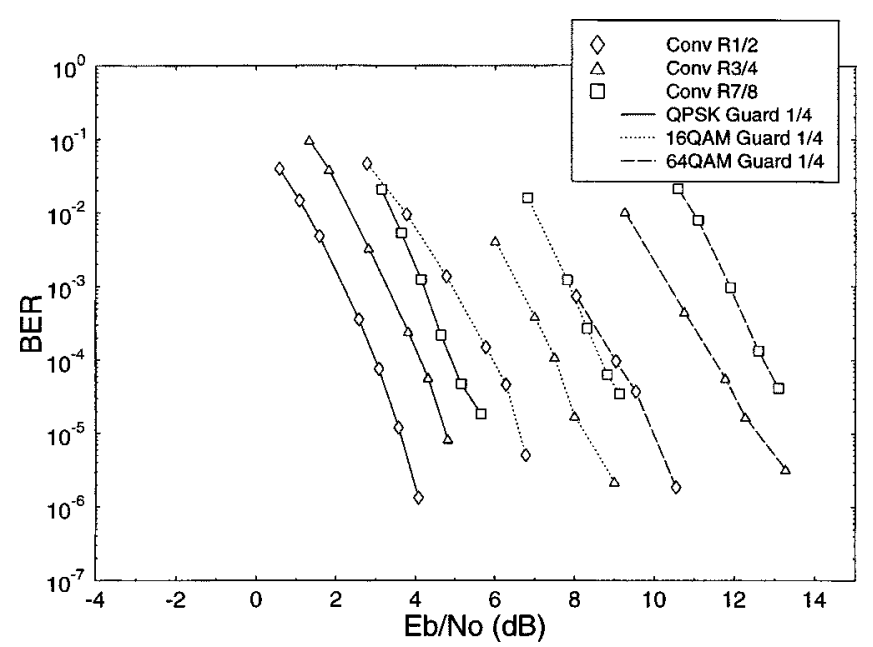

(a)

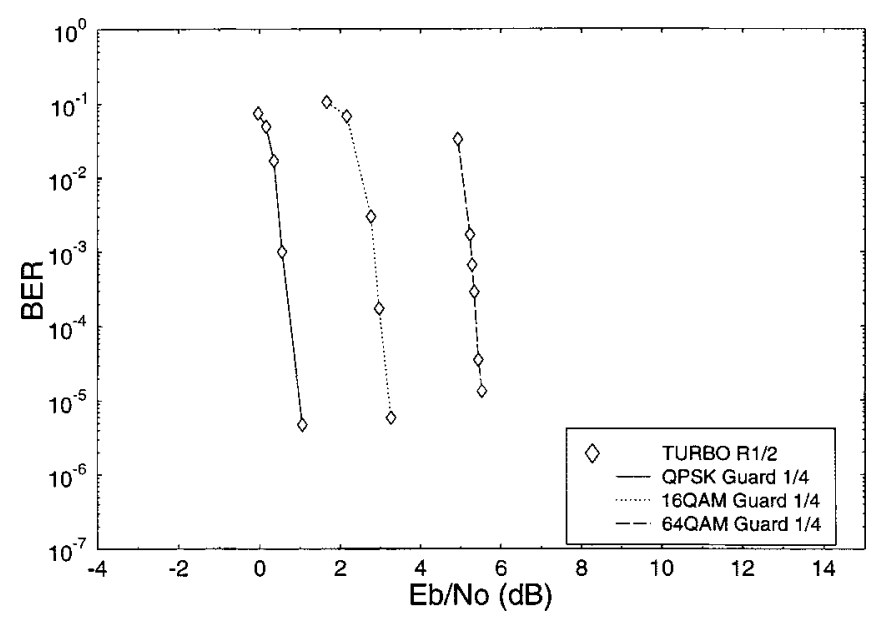

(b)

Fig. 22. BER after (a) convolutional decoding and (b) turbo decoding for the DVB-T scheme over stationary, nondispersive AWGN channels for nonhierarchical transmission.

QPSK modulation mode and a 1/2 coding rate, the turbo code required an approximately $5.5 \mathrm{~dB}$ lower channel SNR for maintaining near-unimpaired video quality than the convolutional code.

Comparing Fig. 23(a) and Fig. 24(a), we note that the Reed-Solomon decoder becomes effective in lowering the bit error probability of the transmitted data further below the BER threshold of $10^{-4}$. From these figures we also observe that the rate-3/4 convolutional code is unsuitable for transmission over the highly dispersive hilly terrain channel used in this experiment, when 64-QAM is employed. When the rate-7/8 convolutional code is used, both the 16-QAM and 64-QAM schemes perform poorly. As for the QPSK modulation scheme, a convolutional code rate as high as $7 / 8$ can still provide a satisfactory performance after Reed-Solomon decoding.

In conclusion, Tables VII and VIII summarize the system performance in terms of the channel SNR (CSNR) required for maintaining less than $2 \mathrm{~dB}$ PSNR video degradation. It was observed that at this PSNR degradation decoding errors were still perceptually unnoticeable to the viewer due to the 30 frames/s refresh-rate, although the typical still-frame shown in Fig. 27 in this scenario exhibits some degradation. It is important to un-

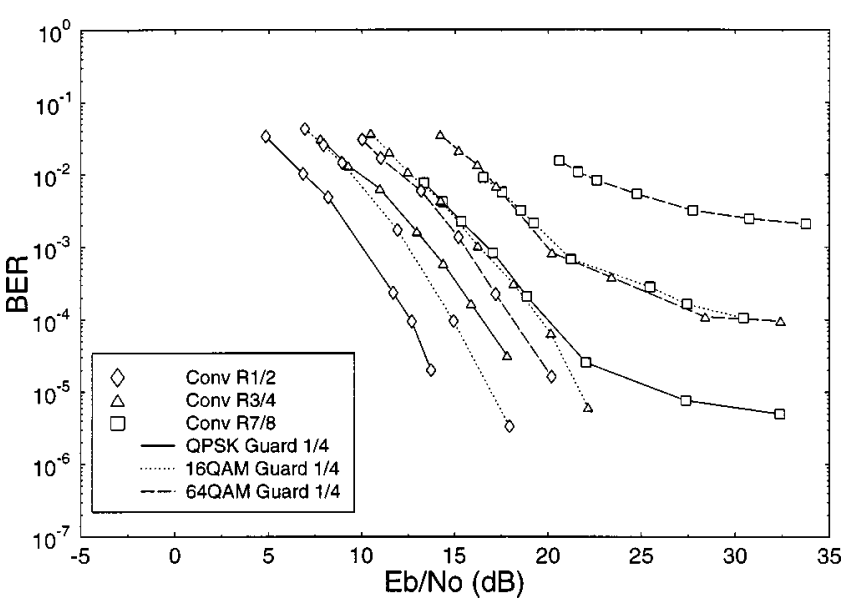

(a)

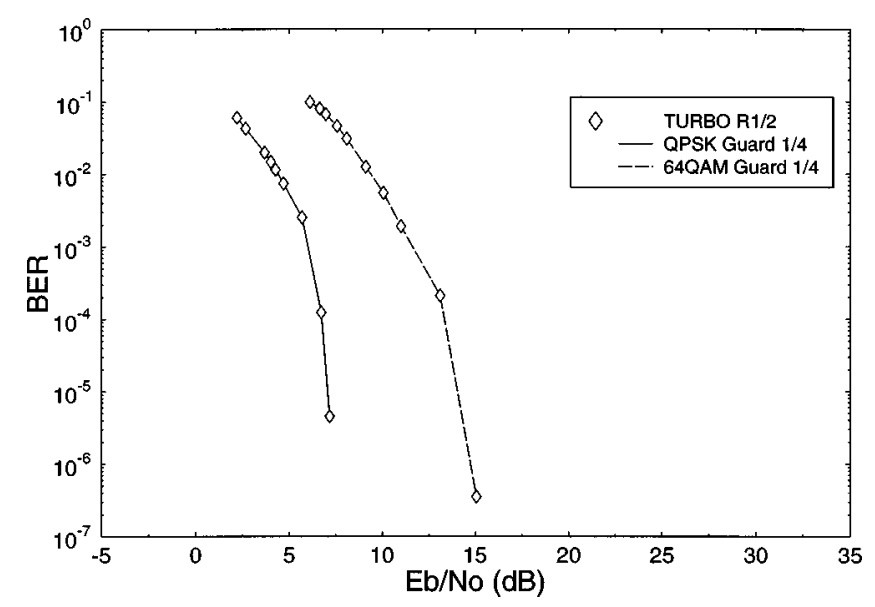

(b)

Fig. 23. BER after (a) convolutional decoding and (b) turbo decoding for the DVB-T scheme over the wideband fading channel of Fig. 11 for nonhierarchical transmission.

derline once again that the $K=3$ turbo code and the $K=$ 7 convolutional code exhibited comparable complexities. The higher performance of the turbo codec facilitates for example the employment of turbo-coded 16-QAM at a similar channel SNR, where convolutional-coded QPSK can be invoked. This in turn allows us to double the bit rate within the same bandwidth and hence improve the video quality. In Section VIII, we shall present the results of our investigations employing the DVB-T system in a hierarchical transmission scenario.

\section{Performance of The DVB TERRESTRIAl Scheme EMPLOYING HIERARCHICAL TRANSMISSION}

The philosophy of the hierarchical transmission mode is that the natural BER difference of a factor 2 to 3 of the 16-QAM modem is exploited for providing unequal error protection for the FEC-coded video streams B3 and B4 of Fig. 13 [6]. If the sensitivity of the video bits requires a different BER ratio between the B3 and B4 streams, the choice of the FEC codes protecting the video streams B1 and B2 of Fig. 13 can be appropriately adjusted to equal out or to augment these differences.

Below we will invoke the DVB-T hierarchical scheme in a mobile broadcasting scenario. We shall also demonstrate the improvements which turbo codes offer, when replacing the 


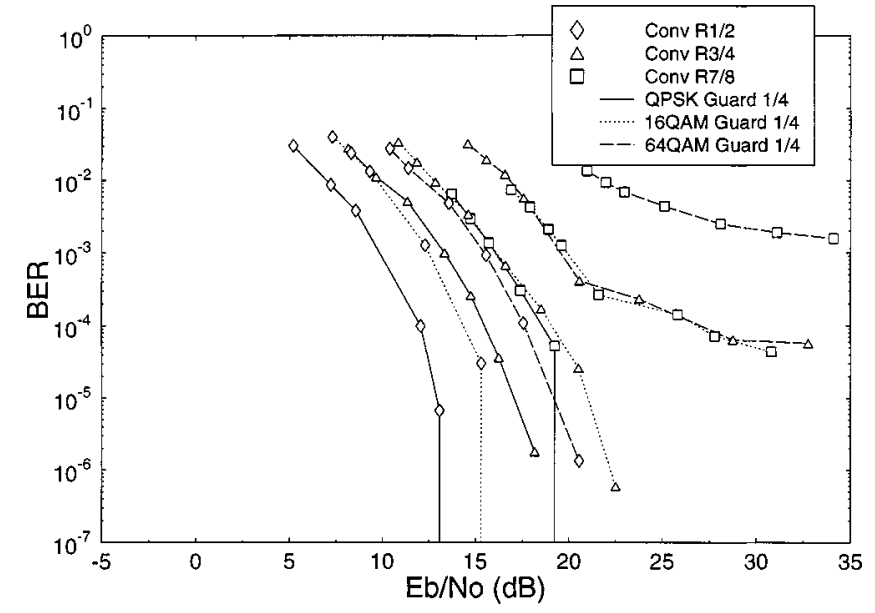

(a)

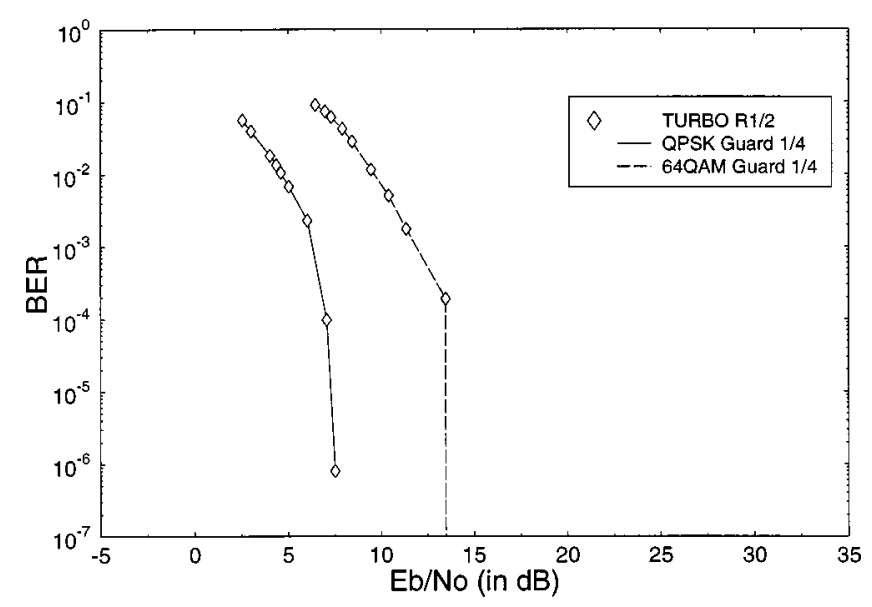

(b)

Fig. 24. BER after (a) RS and convolutional decoding and (b) RS and turbo decoding for the DVB-T scheme over the wideband fading channel of Fig. 11 for nonhierarchical transmission.

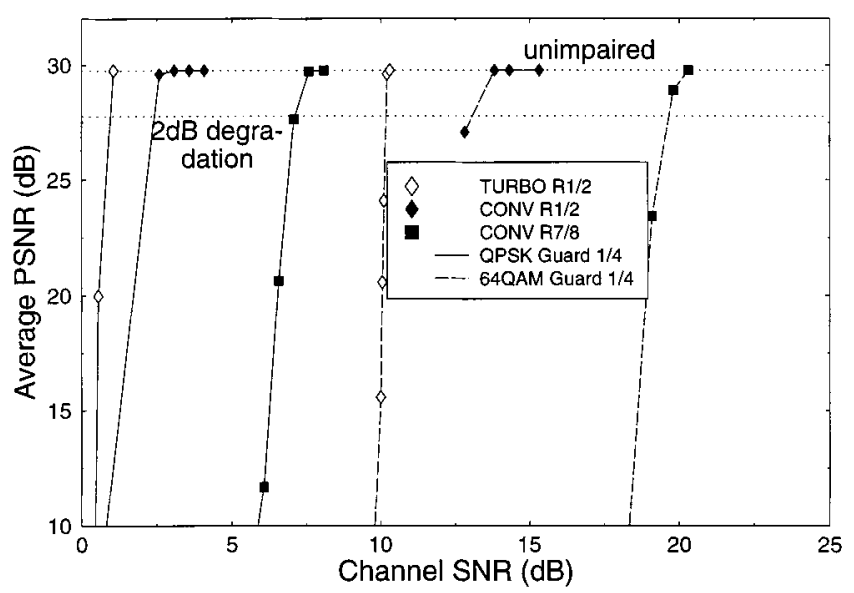

Fig. 25. Average PSNR versus channel SNR of the DVB scheme [25] over nondispersive AWGN channels for nonhierarchical transmission.

tected by the rate $3 / 4$ and $7 / 8$ codes was that when the low-priority partition data was corrupted, the error-free high-priority data available was insufficient for concealing the errors, as discussed in Section VI. We have also experimented with the combination of rate $2 / 3$ convolutional coding and rate $1 / 2$ convolu-
TABLE VIII

Summary of the Non-Hierchical Performance Results Over Wideband Fading Channels Tolerating a PSNR DEgRADATION OF $2 \mathrm{~dB}$ THE BER MEASURE REFERS TO BER AFTER VITERBI OR TURBo DECODING.

\begin{tabular}{|l|l|r|r|r|}
\hline Mod. & Code & $\begin{array}{r}\text { CSNR } \\
(\mathrm{dB})\end{array}$ & $E_{b} / N_{0}$ & BER \\
\hline QPSK & Turbo (1/2) & 6.63 & 6.63 & $2.5 \cdot 10^{-4}$ \\
\hline 64QAM & Turbo (1/2) & 15.82 & 11.05 & $2 \cdot 10^{-3}$ \\
\hline QPSK & Conv $(1 / 2)$ & 10.82 & 10.82 & $6 \cdot 10^{-4}$ \\
\hline 64QAM & Conv $(1 / 2)$ & 20.92 & 16.15 & $7 \cdot 10^{-4}$ \\
\hline QPSK & Conv $(7 / 8)$ & 20.92 & 18.49 & $3 \cdot 10^{-4}$ \\
\hline
\end{tabular}

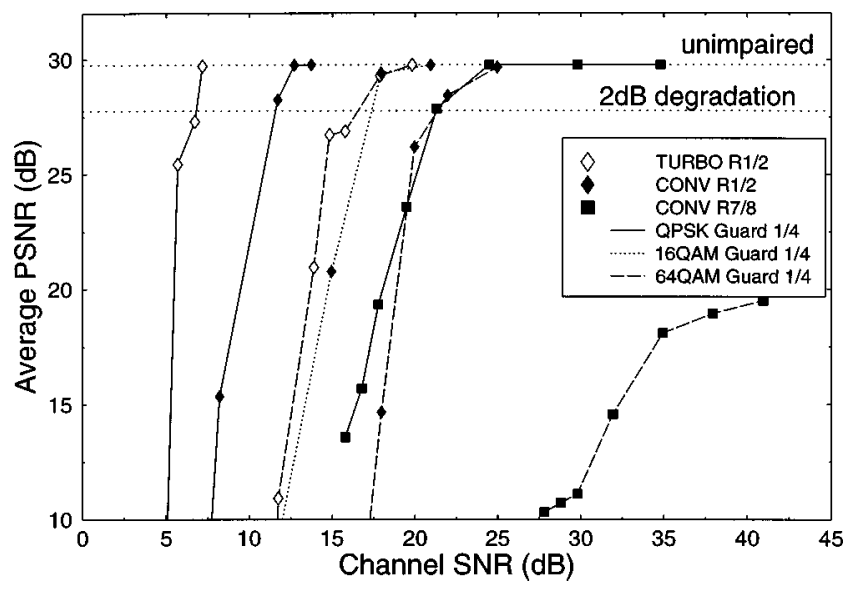

Fig. 26. Average PSNR versus channel SNR of the DVB scheme [1] over the wideband fading channel of Fig. 11 for nonhierarchical transmission.

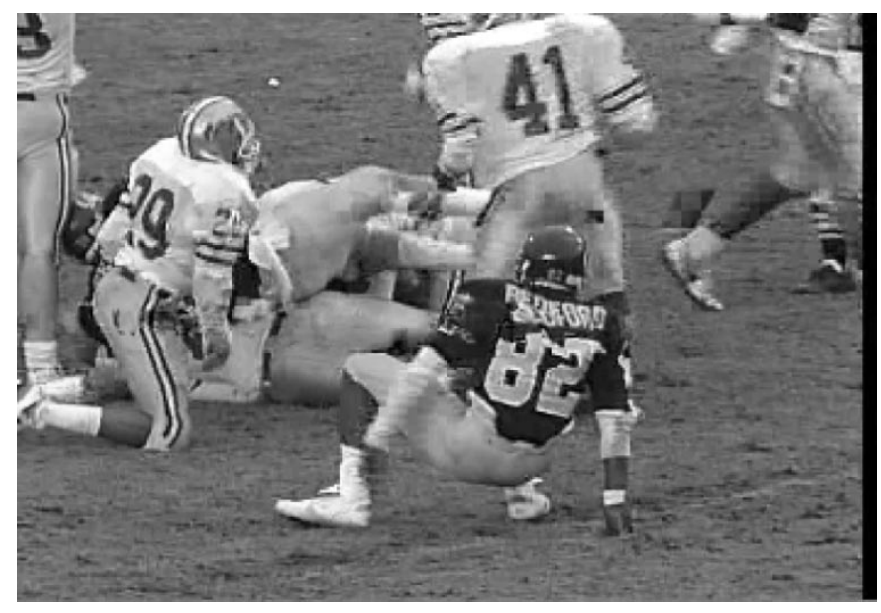

Fig. 27. Frame 79 of "Football" sequence, which illustrates the visual effects of minor decoding errors at a BER of $2.10^{-4}$ after convolutional decoding. The PSNR degradation observed is approximately $2 \mathrm{~dB}$. The sequence was coded using a rate-7/8 convolutional code and transmitted emplying QPSK modulation.

tional coding, in order to protect the high- and low-priority data, respectively. From Fig. 30(a) we observed that the performance of this 2/3- and 1/2-rate combination approached that of the rate $1 / 2$ convolutional code in both partitions. This was expected, since now more data can be inserted into the high-priority partition. Hence, in the event of decoding errors in the low-priority data we had more error-free high-priority data that can be used to reconstruct the received image. 


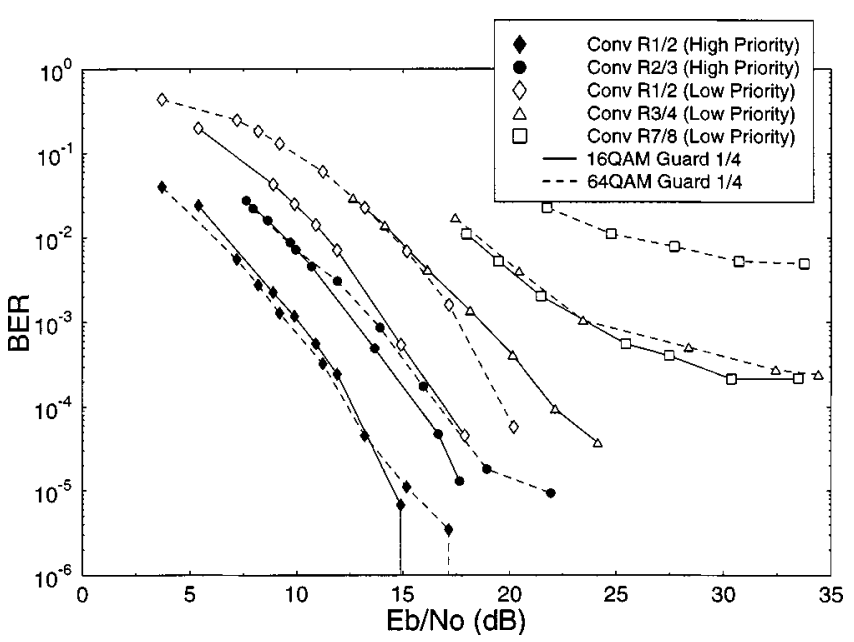

(a)

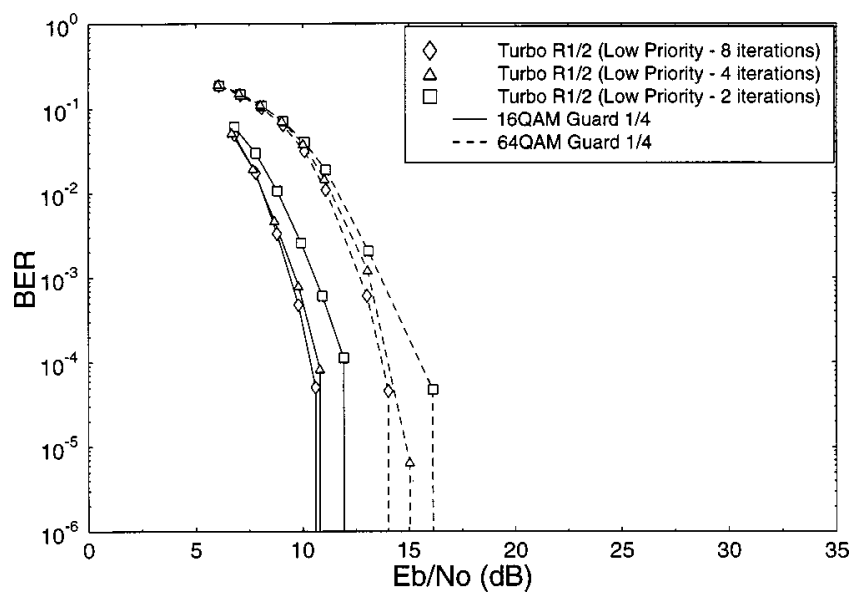

(b)

Fig. 28. BER after (a) convolutional decoding and (b) turbo decoding for the DVB-T hierarchical scheme over the wideband fading channel of Fig. 11 using the schematic of Fig. 13 as well as Algorithms 1 and 2. In (b), the BER of the turbo or convolutional-coded high-priority partition is not shown.

Our last combination investigated involved using rate $1 / 2$ turbo coding and convolutional coding for the high- and low-priority partitions, respectively. Comparing Figs. 31 and 30(a), the channel SNR required for achieving unimpaired video transmission in both cases were similar. This was expected, since the turbo-convolutional combination's video performance is dependent on the convolutional code's performance in the low-priority partition.

Lastly, comparing Figs. 30 and 26, we found that the unimpaired PSNR condition was achieved at similar channel SNR's for the hierarchical and nonhierarchical schemes, suggesting that the data partitioning scheme had not provided sufficient performance improvements in the context of the mobile DVB scheme to justify its added complexity. Again, this was a consequence of relegating a high proportion of video bits to the low-integrity partition.

\section{CONClusions AND FutURe WORK}

In this contribution, we have investigated the performance of a turbo-coded DVB system in a mobile environment. A range of system performance results was presented based on the standard

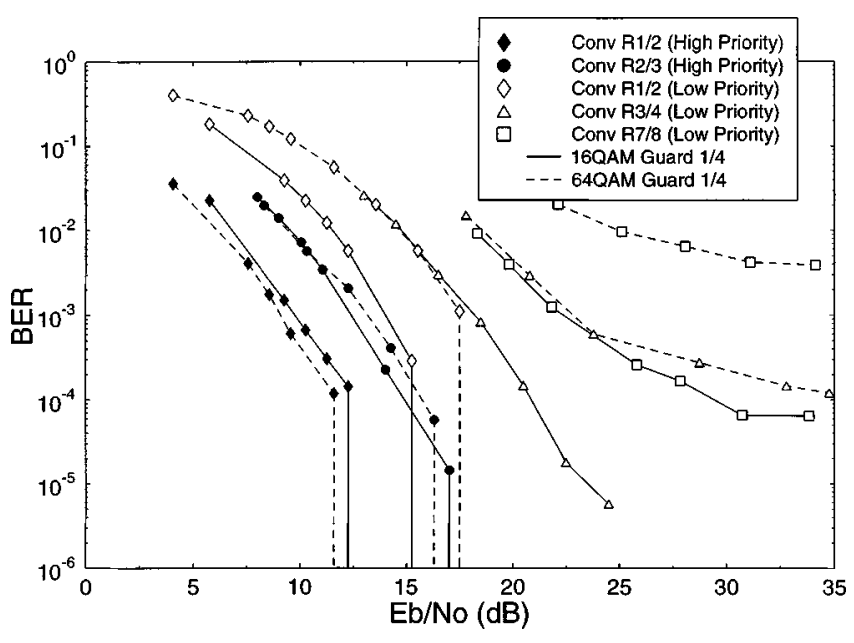

(a)

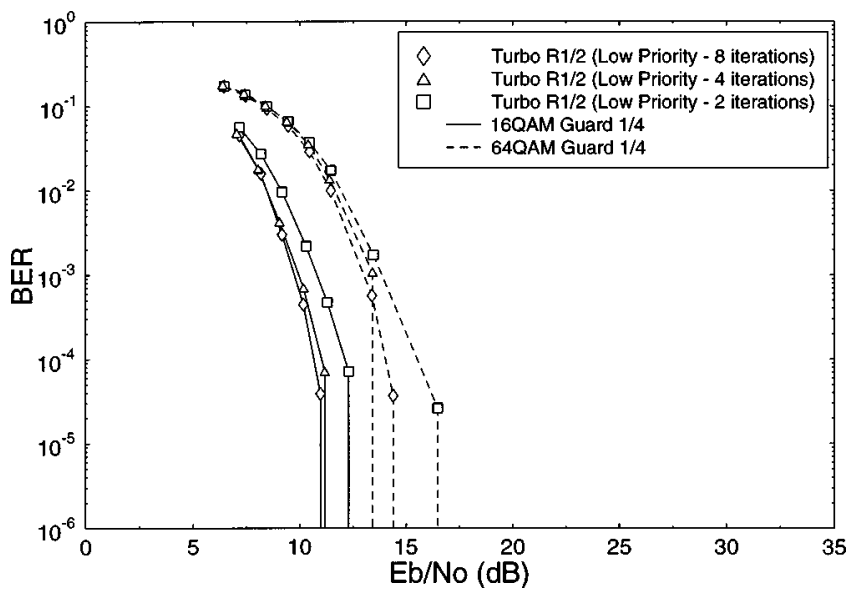

(b)

Fig. 29. BER after (a) RS and convolutional decoding and (b) RS and turbo decoding for the DVB-T hierarchical scheme over the wideband fading channel of Fig. 11 using the schematic of Fig. 13 as well as Algorithms 1 and 2. In (b), the BER of the turbo or convolutional-coded high-priority partition is not shown.

DVB-T scheme as well as on an improved turbo-coded scheme. The convolutional code specified in the standard system was replaced by turbo coding, which resulted in a substantial coding gain of around $5 \mathrm{~dB}$. It is important to underline once again that the $K=3$ turbo code and the $K=7$ convolutional code exhibited comparable complexities. The higher performance of the turbo codec facilitates, for example, the employment of turbocoded 16-QAM at a similar SNR, where convolutional-coded QPSK can be invoked. This in turn allows us to double the video bit rate within the same bandwidth and hence to improve the video quality. We have also applied data partitioning to the MPEG-2 video stream to gauge its efficiency in increasing the error resilience of the video codec. However, from these experiments we found that the data partitioning scheme did not provide substantial improvements compared to the nonpartitioned video transmitted over the nonhierarchical DVB-T system. Our future work will be focused on extending this DVB-T system study to incorporate various types of channel models, as well as on investigating the effects of different Doppler frequencies on the system. Further work will also be dedicated to trellis coded 


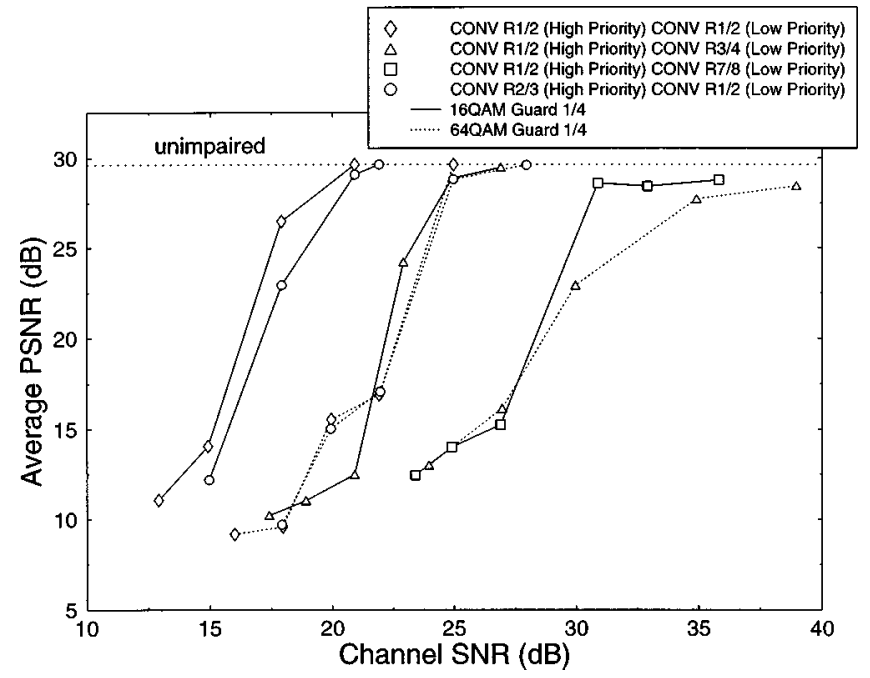

(a)

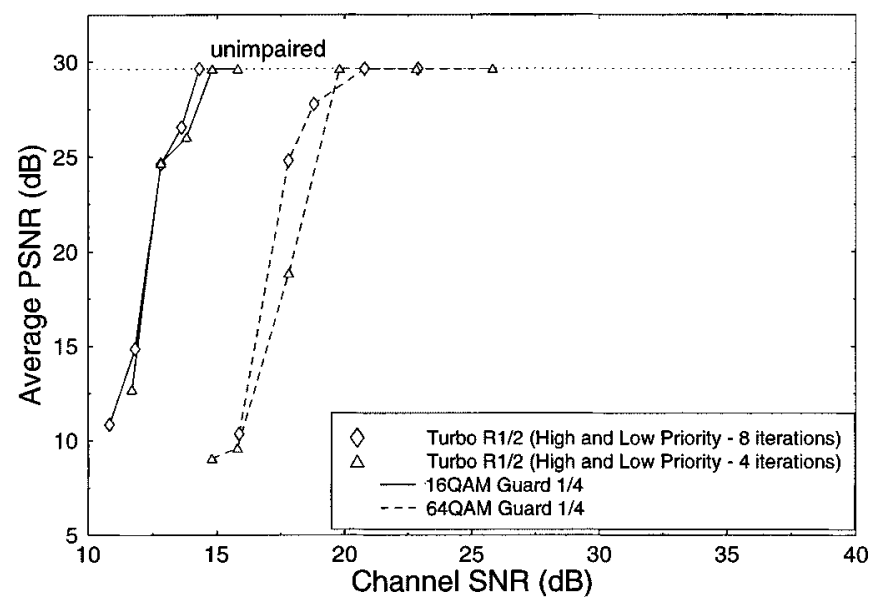

(b)

Fig. 30. Average PSNR versus channel SNR for (a) standard DVB scheme [1] and (b) system with turbo coding employed in both partitions, for transmission over the wideband fading channel of Fig. 11 for hierarchical transmission using the schematic of Fig. 13 as well as Algorithms 1 and 2.

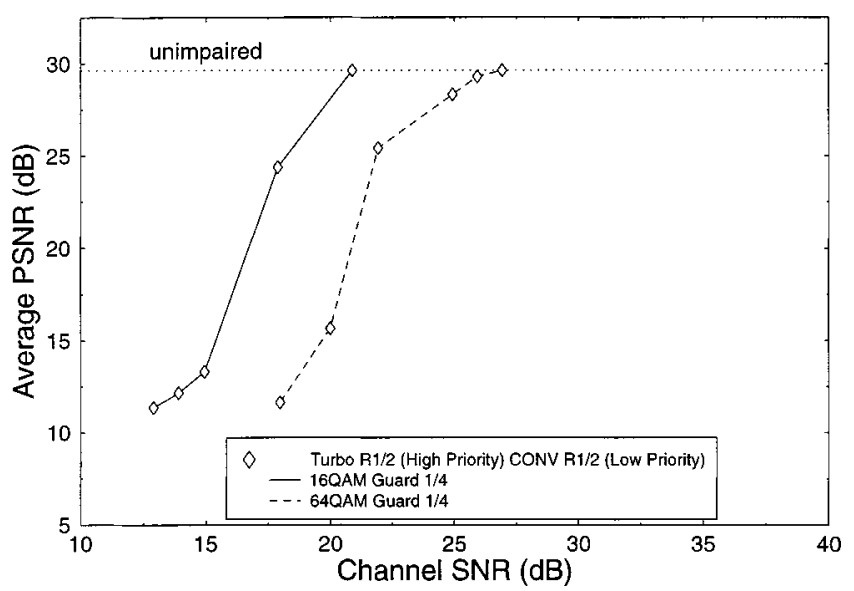

Fig. 31. Average PSNR versus channel SNR of the DVB scheme, employing turbo coding in the high-priority partition and convolutional coding in the low-priority partition, over the wideband fading channel of Fig. 11 for hierarchical transmission using the schematic of Fig. 13 as well as Algorithms 1 and 2.

modulation (TCM) and turbo trellis coded modulation (TTCM) based OFDM. The impact of employing various types of turbo interleavers on the system performance is also of interest. A range of further wireless video communications issues are addressed in [26], [27].

\section{ACKNOWLEDGMENT}

The authors would like to thank the reviewers for the constructive comments.

\section{REFERENCES}

[1] ETSI, "Digital video broadcasting (DVB-T); Framing structure, channel coding and modulation for digital terrestrial television,", EN 300744 Vl.1.2, August 1997.

[2] ETSI, "Digital video broadcasting (DVB-C); Framing structure, channel coding and modulation for cable systems,", EN 300429 V1.2.1, December 1997.

[3] ETSI, "Digital video broadcasting (DVB-S); Framing structure, channel coding and modulation for 11/12 GHz satellite services,", EN 300421 Vl.1.2, August 1997.

[4] R. Steele and L. Hanzo, Eds., Mobile Radio Communications, 2 ed: John Wiley \& Sons and IEEE Press, 1999

[5] A. M. Michelson and A. H. Levesque, Error Control Techniques for Digital Communication: Wiley Interscience, 1985.

[6] L. Hanzo, W. Webb, and T. Keller, Single and Multicarrier Quadrature Amplitude Modulation: John Wiley \& Sons and IEEE Press, April 2000.

[7] S. O'Leary and D. Priestly, "Mobile broadcasting of DVB-T signals," IEEE Transactions on Broadcasting, vol. 44, pp. 346-352, September 1998.

[8] W.-C. Lee, H.-M. Park, K.-J. Kang, and K.-B. Kim, "Performance analysis of Viterbi decoder using channel state information in COFDM system," IEEE Transactions on Broadcasting, vol. 44, pp. 488-496, December 1998.

[9] S. O'Leary, "Hierarchical transmission and COFDM systems," IEEE Transactions on Broadcasting, vol. 43, pp. 166-174, June 1997.

[10] L. Thibault and M. T. Le, "Performance evaluation of COFDM for digital audio broadcasting -Part I: Parametric study," IEEE Transactions on Broadcasting, vol. 43, pp. 64-75, March 1997.

[11] B. G. Haskell, A. Puri, and A. N. Netravali, Digital Video: An Introduction To MPEG-2, ser. Digital Multimedia Standards Series: Chapman \& Hall, 1997.

[12] "Information Technology - Generic Coding of Moving Pictures and Associated Audio Information-Part 2: Video,", ISO/IEC 13 818-2, March 1995.

[13] L. Hanzo and J. Woodard, "An intelligent cordless voice terminal for indoors communications," IEEE Transactions on Vehicular Technology, vol. 44, pp. 735-749, November 1995.

[14] L. Hanzo and J. Streit, "Adaptive low rate wireless videophone schemes," IEEE Transactions on Circuits and Systems for Video Technology, vol. 5, pp. 305-318, August 1995.

[15] K. Rao and P. Yip, Discrete Cosine Transform. New York, NY: Academic Press, 1990.

[16] P. Shelswell, "The COFDM modulation system: The heart of digital audio broadcasting," Electronics \& Communication Engineering Journal, vol. 7, pp. 127-136, June 1995.

[17] C. Berrou, A. Glavieux, and P. Thitimajshima, "Near Shannon limit error-correcting coding and decoding: Turbo codes," in IEEE Proceedings of the International Conference on Communications, Geneva, Switzerland, May 1993, pp. 1064-1070.

[18] C. Berrou and A. Glavieux, "Near optimum error correcting coding and decoding: Turbo codes," IEEE Transactions on Communications, vol. 44, pp. 1261-1271, October 1996.

[19] S. B. Wicker, Error Control Systems for Digital Communication and Storage: Prentice Hall, 1994.

[20] A. Barbulescu and S. Pietrobon, "Interleaver design for turbo codes," IEE Electronics Letters, pp. 2107-2108, December 1994.

[21] P. Robertson, E. Villebrun, and P. Hoeher, "A comparison of optimal and suboptimal MAP decoding algorithms operating in the log domain," in IEEE Proceedings of the International Conference on Communications, June 1995, pp. 1009-1013.

[22] M. Failli, "Digital land mobile radio communications COST 207," European Commission, tech. rep., 1989.

[23] H. Gharavi and S. Alamouti, "Multipriority video transmission for thirdgeneration wireless communication system," Gharavi and Hanzo [27], pp. 1751-1763. 
[24] A. Aravind, M. R. Civanlar, and A. R. Reibman, "Packet loss resilience of MPEG-2 scalable video coding algorithms," IEEE Transactions on Circuits and Systems for Video Technology, vol. 6, pp. 426-435, October 1996.

[25] M. Ghanbari and V. Seferidis, "Cell-loss concealment in ATM video codecs," IEEE Transactions on Circuits and Systems for Video Technology, vol. 3, p. 1993, June 1993.

[26] L. Hanzo, P. J. Cherriman, and J. Streit, Modern Video Compression and Communications: Principles and Applications for Fixed and Wireless Channels: IEEE Press, 2000. In preparation.

[27] H. Gharavi and L. Hanzo, eds., Special Issue on Video Transmission for Mobile Multimedia Applications, vol. 87, October 1999.

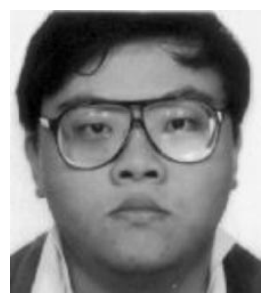

Chee-Siong Lee studied electrical engineering at the University of Southampton and graduated with the First Class Honors Degree in 1997. He was also awarded the top prize of his class. Since then, he has been with the Wireless Multimedia Communications Group at the University of Southampton, working toward the Ph.D. degree in mobile multimedia communications. His current areas of interest include video compression and communications.

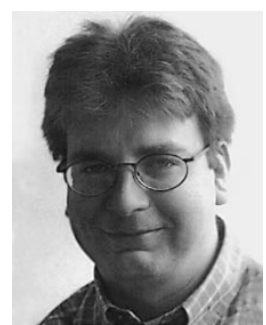

Thomas Keller studied electrical engineering at the University of Karlsruhe, Ecole Superieure d'Ingenieurs en Electronique et Electrotechnique, Paris, and the University of Southampton. He graduated with the Dipl.-Ing. degree in 1995. Since then, he has been with the Wireless Multimedia Communications Group at the University of Southampton, working toward the Ph.D. degree in mobile communications. His current areas of interest include adaptive OFDM transmission, wideband channel estimation, and error correction coding

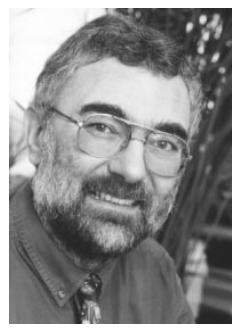

Lajos Hanzo has held various research and academic posts in Hungary, Germany, and the UK during his 24-year career in telecommunications. Since 1986, he has been a Member of the Academic Staff of the Department of Electronics and Computer Science, University of Southampton, UK and has been a Consultant to Multiple Access Communications Ltd., UK. Currently, he holds the Chair in Telecommunications. As a member of a multinational consortium and funded by the European Community as well as by the Engineering and Physical Sciences Research Council (EPSRC) UK, he is currently conducting research toward the next generation of wireless multimedia systems. He published widely in Wireless Multimedia Communications, including three monographs and more than 200 research papers, organized and chaired conference sessions, presented overview lectures and was awarded a number of distinctions. He manages an academic research team. He is a Member of the IEE and Senior Member of the IEEE. For further information please refer to http://www-mobile.ecs.soton.ac.uk. 